Interfaces and Free Boundaries 15 (2013), 281-322

DOI 10.4171/IFB/304

\title{
Regularity of expanding front and its application to solidification/melting in undercooled liquid/superheated solid
}

\author{
XINFU CHEN \\ Department of Mathematics, University of Pittsburgh, Pittsburgh, PA 15260, USA \\ E-mail: xinfu@pitt.edu \\ HUIQIANG JIANG \\ Department of Mathematics, University of Pittsburgh, Pittsburgh, PA 15260, USA \\ E-mail: hqjiang@pitt.edu
}

[Received 3 February 2012 and in revised form 14 May 2013]

\begin{abstract}
This article proves that fronts of expanding domains with Hölder continuous speeds are contained in finite unions of Lipschitz graphs. As an application, the global in time existence of a solution to a free boundary problem modelling solidification in undercooled liquid or liquidation in superheated solid is established; here the propagation speed of the liquid/solid interface is assumed to be a known positive smooth function of the temperature, known as a kinetic undercooling/superheating effect.
\end{abstract}

2010 Mathematics Subject Classification: Primary 35R35; Secondary 35Q35, 35B65, 80A20.

Keywords: Moving front, regularity, solidification, melting, undercooling, superheating

\section{Introduction}

Consider a geometric problem of domain expansion with a given speed $v$ starting from a given bounded initial shape $\mathrm{D}_{0} \subset \mathbb{R}^{n}(n \geqslant 1)$. It is to find at any time $t \geqslant 0$ the shape $\mathrm{D}(t)$ of the evolving domain, with the expansion law that its boundary $\Gamma(t):=\partial \mathrm{D}(t)$ moves outward with speed $v$.

Using level sets [3, 12, 18, 22, 26, 29, 33], this problem can be modeled by the Hamilton-Jacobi equation

$$
\phi_{t}(x, t)+v(x, t)|\nabla \phi(x, t)|=0, \quad(x, t) \in \mathbb{R}^{n} \times(0, \infty)
$$

where $\mathrm{D}(t)$ is defined as the closed set $\left\{x \in \mathbb{R}^{n}: \phi(x, t) \leqslant 0\right\}$. The front $\Gamma(t)=\partial \mathrm{D}(t)$ is (contained in) the zero-level set of $\phi$ so it advances with normal velocity

$$
v=\frac{-\phi_{t}}{|\nabla \phi|} \text {. }
$$

From a geometric point of view, the Hamilton-Jacobi equation cannot have a smooth solution except for special $v$ and $\mathrm{D}_{0}$ since in general characteristic curves intersect thereby forming singularities. For this, viscosity solutions are introduced and have been successfully used; see [1, 4-12, 14-16, $18,22,24,26,29]$ and the references therein. When $v$ changes sign, even if it is smooth and even if the viscosity solution is unique, the zero-level set $\{x: \phi(x, t)=0\}$ may contain open sets (marshy regions) thereby causing troubles in solving many free boundary problems; see Giga, Goto, and Ishii [22] for an example of a treatment for such troubles. Here we restrict to $v$ positive, so the domain is truly expanding and marshy regions should not exist. When $v$ is Lipschitz continuous 
in its space variable, Barles, Cardaliaguet, Ley and Monteillet [4] proved the uniqueness of the viscosity solution. Moreover, viscosity solutions are stable under perturbations of the speed and the initial set. When $v$ is only Hölder continuous, such a stability may be lost.

Introduce arriving time $\tau(x)$ as the time that the expanding front moves to the position $x$. Then $\Gamma(t)$ is the $t$-level set of $\tau$ and, with $\phi(x, t)=\tau(x)-t$, the above Hamilton-Jacobi equation becomes

$$
v(x, \tau(x))|\nabla \tau(x)|=1 \quad \forall x \in \mathbb{R}^{n} \backslash \mathrm{D}_{0}, \quad \tau(x)=0 \text { on } \mathrm{D}_{0} .
$$

Instead of using partial differential equations, we use the Lax formula to define our solution:

$$
\mathrm{D}(t):=\left\{\mathbf{x}(t): \mathbf{x} \in \operatorname{Lip}\left([0, t] ; \mathbb{R}^{n}\right), \quad \mathbf{x}(0) \in \mathrm{D}_{0}, \quad|\dot{\mathbf{x}}| \leqslant v(\mathbf{x}, \cdot) \text { a.e. in }(0, t)\right\} .
$$

Here Lip $\left([0, t] ; \mathbb{R}^{n}\right)$ is the space of Lipschitz continuous functions from $[0, t]$ to $\mathbb{R}^{n}$. The geometric meaning of such a solution is clear: $\mathrm{D}(t)$ is the set that can be reached at time $t$ by a particle starting from $\mathrm{D}_{0}$ and traveling with a speed no bigger than $v$. Such defined solution can be shown to be identical to that derived from viscosity solutions of the Hamilton-Jacobi equations for $\phi$ or for $\tau$.

The main purpose of this paper is to study regularity of the front $\Gamma(t):=\partial \mathrm{D}(t)$ where $\mathrm{D}(t)$ is defined as in (1.1) and $v$ is positive and Hölder continuous: for some constants $0 \leqslant \alpha \leqslant 1, c \geqslant 1$ and $\kappa \geqslant 1$,

$$
1 \leqslant v(x, t) \leqslant c, \quad|v(x, t)-v(y, t)| \leqslant \kappa^{\alpha}|x-y|^{\alpha} \quad \forall x, y \in \mathbb{R}^{n}, t \geqslant 0 .
$$

Note that as long as $v$ has a positive lower bound, a proper time unit change can make the lower bound of $v$ the unit 1 as above. Our main result is following:

THEOREM 1 Each front $\Gamma(t)$ is contained in a union of finitely many Lipschitz graphs. The area, measured by $\mathcal{H}^{n-1}$, of $\Gamma(t)$ in a ball

$$
B(x, \rho):=\left\{y \in \mathbb{R}^{n}:|x-y|<\rho\right\}
$$

satisfies

$$
\mathcal{H}^{n-1}(\Gamma(t) \cap B(x, \rho)) \leqslant C\left(n, \alpha, c, \mathrm{D}_{0}\right) \rho^{n-1} \quad \forall x \in \mathbb{R}^{n}, \rho \in\left(0, \kappa^{-1}\right] .
$$

One can check that the estimate is invariant under the scaling change $(x, t) \longrightarrow(\hat{x}, \hat{t}):=$ $(\kappa x, \kappa t)$. Such an estimate, with $\kappa^{-1}$ being replaced by $\kappa^{\alpha^{2}-2}$ and in the two dimensional case, is discovered by Su and Burger in $[34,35]$ where many original ideas were introduced. One of their novelties is to study a boundary characteristic trajectory defined as

$$
\mathbf{x}([0, t]):=\{\mathbf{x}(s): 0 \leqslant s \leqslant t\}
$$

where $|\dot{\mathbf{x}}(s)|=v(\mathbf{x}(s), s)$ and $\mathbf{x}(s) \in \Gamma(s)$ for every $s \in[0, t]$. They proved that the trajectory is microscopically almost straight; more precisely, the length of any tiny segment of the trajectory is bounded by $l+O\left(l^{1+\alpha}\right)$ where $l$ is the line distance between end points of the segment. With original and involving two dimensional arguments, they are able to claim their version of (1.2).

Here we shall consider general space dimension $n \geqslant 1$. We improve Su and Burger's estimate by showing that any boundary characteristic trajectory is microscopically straight in the sense that $\dot{\mathbf{x}}(s)=\mathbf{n}(s) v(\mathbf{x}(s), s)$ where the direction unit vector function $\mathbf{n}(\cdot)$ is Hölder continuous on $[0, t]$ :

$$
\left|\mathbf{n}\left(t_{1}\right)-\mathbf{n}\left(t_{2}\right)\right| \leqslant \frac{15 c^{\beta / 2}}{1-2^{-\beta}} \kappa^{\beta}\left|t_{1}-t_{2}\right|^{\beta} \quad \forall t_{1}, t_{2} \in[0, t] \quad\left(\beta:=\frac{\alpha}{2-\alpha}\right) .
$$


The Hölder exponent $\beta$ is optimal. It improves the estimate for the length of trajectory from $\mathrm{Su}$ and Burger's $l+O\left(l^{\alpha}\right)$ to its optimal $l+O\left(l^{1+2 \beta}\right)$. Similar estimate has also been established in [8] with Hölder exponent $\frac{\alpha}{2}$ under a different setting. The most significant application of this new estimate is that it allows us to construct efficiently comparison particle motion paths to show that $\Gamma(s)$ near $\mathbf{x}(s)$ is sandwiched between two $C^{1+\alpha / 2}$ graphs touching at $\mathbf{x}(s)$, for each $s \in(0, t)$; technically, writing $p=\mathbf{x}(s)$ and $\mathbf{n}=\mathbf{n}(s)$, we can prove the following inclusions:

$$
\begin{gathered}
\left\{z \in B\left(p, \kappa^{\beta} s^{1+\beta}\right):(z-p) \cdot \mathbf{n} \leqslant-C \kappa^{\frac{\alpha}{2}}|z-p|^{1+\frac{\alpha}{2}}\right\} \subset \mathrm{D}(s) \quad \forall s \in(0, t], \\
\left\{z \in B\left(p, \kappa^{\beta}|t-s|^{1+\beta}\right):(z-p) \cdot \mathbf{n}>C \kappa^{\frac{\alpha}{2}}|z-p|^{1+\frac{\alpha}{2}}\right\} \subset \mathbb{R}^{n} \backslash \mathrm{D}(s) \quad \forall s \in[0, t)
\end{gathered}
$$

where $C=C(n, c, \alpha)$ is a constant depending only on $n, c$ and $\alpha$. Here again, the exponent $1+\alpha / 2$ is optimal. Using both inclusions, we immediately see that

$$
\mathbf{n}(s):=\frac{\dot{\mathbf{x}}(s)}{|\dot{\mathbf{x}}(s)|}
$$

is the unit outward normal to $\mathrm{D}(s)$ at $\mathbf{x}(s)$, for each $s \in(0, t)$. Using only (1.3) at $s=t$, we can derive that in a small ball, $\Gamma(t)$ is contained in a union of at most $2 n$ (one in each positive and negative coordinate direction) graphs of the form, after rotation, $x_{1}=\psi\left(x_{2}, \cdots, x_{n}\right)$ where $\psi$ is Lipschitz continuous with Lipschitz constant no bigger than $2 \sqrt{n}$. The size of the ball for such a result to hold depends on $t$. To make it non-diminishing as $t \searrow 0$, we assume that $\mathrm{D}_{0}$ satisfies the following interior cone condition:

At every $q \in \partial D_{0}, D_{0}$ contains a cone with vertex $q$ and fixed size and open angle.

Under this assumption, we show that the radii of the balls (for $\Gamma(t)$ being in union of finite graphs) can be set at a uniform size $\propto \kappa^{-1}$. From this, estimate (1.2) follows.

Interesting results for this problems have also been obtained in different settings by Cannarsa and Cardaliaguet in [7] where it is shown that the expanding domain has finite perimeter and Alvarez, Cardaliaguet and Monneau in [1] where existence of global weak solutions is studied. Their methods of proof, based on an interior sphere property of the attainable sets, are totally different from ours, and they also require somewhat stronger condition on expanding speed and initial shape. Recently, similar regularity results have also been obtained by Cardaliaguet, Ley and Mointeiliet [8] where in addition to the Hölder continuity of the expanding speed, they needed stronger condition

$$
|v(x, t)-v(y, t)| \leqslant C|y-x|(1+|\ln | x-y||) .
$$

As an application of (1.2), we consider a solidification process in crystal growth. Set the latent heat be 1 and denote by $\mathrm{D}(t)$ the region occupied by the solid at time $t$. The conservation of energy gives the heat equation for the temperature $u$ :

$$
\frac{\partial}{\partial t}\left(u-\chi_{\mathrm{D}(t)}\right)=\Delta u-f \quad \text { in } \mathbb{R}^{n} \times(0, \infty)
$$

Here $f$ is a given function representing the absorption of heat released from solidification and

$$
\chi_{\mathrm{D}(t)}(x):=1 \text { if } x \in \mathrm{D}(t), \quad \chi_{\mathrm{D}(t)}(x):=0 \text { if } x \in \mathbb{R}^{n} \backslash \mathrm{D}(t) .
$$

In a classical formulation the temperature at the solid-liquid interface is assumed to be a constant, say, 0 , called the melting temperature. Then $\mathrm{D}(t)=\{x \mid u(x, t) \leqslant 0\}$ and $\chi_{\mathrm{D}(t)}=1-H(u)$ 
where $H(\cdot)$ is the Heaviside function: $H(u)=0$ if $u \leqslant 0$ and $H(u)=1$ if $u>0$. The problem is then the well-studied Stefan free boundary problem; see [17, 19, 27, 31] and the references therein. Nevertheless, for many materials it is observed that temperature at the solid/liquid interface is dynamic; that is, it depends on the propagation speed of the interface (kinetic undercooling or superheating effect) and on the curvature of the interface (the surface tension effect); see for example $[2,13,21,23-25,28,30,32,36]$ and the references therein. Here we shall ignore the surface tension effect and consider only the kinetic undercooling/superheating. In the case of solidification in undercooled liquid we assume that the propagation speed $v$ of solidification is a known function $V(u)$ of temperature $u$. Hence, when $\{\mathrm{D}(t)\}_{t \geqslant 0}$ is the evolution regions of solid and is described by (1.1), the speed $v$ is given by

$$
v=V \circ u \text {. }
$$

We shall assume that $V(\cdot)$ is positive and Lipschitz: For some constants $L$ and $c$ :

$$
1 \leqslant V(u) \leqslant c, \quad\left|V\left(u_{1}\right)-V\left(u_{2}\right)\right| \leqslant L\left|u_{1}-u_{2}\right| \quad \forall u, u_{1}, u_{2} \in \mathbb{R} .
$$

Here the Lipschitz continuity of $V$ is natural. However, the positivity assumption is artificial since there is a critical point beyond which only melting takes place. Nevertheless, if we are studying solidification in undercooled liquid or in an environment in which temperature is reasonably controlled, the above constraint is not unrealistic. Thus we define our problem as follows:

The free boundary problem of solidification is to find $(u,\{\mathrm{D}(t)\})$ satisfying (1.1), (1.4), and (1.5), subject to given initial region of solid, $D_{0}$, the initial temperature distribution, $u(\cdot, 0)$, the heat abortion, $f$, and the law of propagation $V$ that relates temperature $u$ and propagation speed $v=V(u)$.

The liquidation in superheated solid can be similarly considered and is omitted in this paper.

The short time existence of a classical smooth solution to the free boundary problem starting from smooth initial data with $\mathrm{D}_{0}$ close to a ball has been established by Friedman and Velazquez [20]. Global in time existence of a solution with Hölder continuous temperature function in the 2dimensional case is studied by Su and Burger in [34]. Here we establish the global in time existence for any space dimension, following basically the approach of Su and Burger, with simplifications and new ingredients.

The basic idea for global existence is to investigate regularity of the singular layer potential

$$
w(x, t):=\iint_{\mathrm{D}(t) \backslash \mathrm{D}(0)} K(x-y, t-\tau(y)) d y
$$

where

$$
K(z, s):=(4 \pi s)^{-n / 2} e^{-|z|^{2} /(4 s)} .
$$

This is the solution $u$ to (1.4) with $f=0$ and zero initial data. Su and Burger in [34] carried out critical analysis using (1.2) for a potential more complicated than $w$. Revising their idea we find a clean estimate

$$
[w]_{\gamma}:=\sup _{t} \sup _{x \neq y} \frac{|w(x, t)-w(y, t)|}{|x-y|^{\gamma}} \leqslant C\left(n, c, \alpha, \gamma, \mathrm{D}_{0}\right) \kappa^{\gamma / 2} \quad \forall \gamma \in(0,1) .
$$

Here the factor $\kappa^{\gamma / 2}$, instead of $\kappa^{\gamma}$, is the key to global existence. Indeed, suppose we have a Hölder continuous temperature distribution. Set $\kappa^{\alpha}=L[u]_{\alpha}$. Then for $v:=V \circ u,[v]_{\alpha} \leqslant \kappa^{\alpha}$ so that 
$[w]_{\alpha} \leqslant C \kappa^{\alpha / 2}$. Writing $u=u_{0}+w$ where $u_{0}$ is the extension of the initial temperature $u(\cdot, 0)$ from $\mathbb{R}^{n} \times\{0\}$ to $\mathbb{R}^{n} \times[0, \infty)$ via the heat equation $u_{0 t}=\Delta u_{0}-f$, we have

$$
\frac{\kappa^{\alpha}}{L}=[u]_{\alpha} \leqslant\left[u_{0}\right]_{\alpha}+C \kappa^{\alpha / 2} \leqslant\left[u_{0}\right]_{\alpha}+\frac{L C^{2}}{2}+\frac{\kappa^{\alpha}}{2 L} .
$$

This gives an a priori bound on the semi-Hölder norm:

$$
[u]_{\alpha} \leqslant 2\left[u_{0}\right]_{\alpha}+L C^{2}\left(n, c, \alpha, \mathrm{D}_{0}\right) .
$$

In establishing the existence of a solution, we use the Schauder's fixed point theorem. Fix $\alpha \in$ $(0,1)$ and construct a map $\mathbf{T}$ from

$$
\mathbf{X}(\kappa)=\left\{u \mid[u]_{\alpha} \leqslant \kappa^{\alpha} / L\right\}
$$

to itself via the following process:

$$
u \stackrel{(1.5)}{\longrightarrow} v=V \circ u \stackrel{(1.1)}{\longrightarrow}\{D(t)\} \stackrel{(1.4)}{\longrightarrow} \quad \mathbf{T} u:=u_{0}+w .
$$

Since $\mathbf{T} u \in C^{\gamma, \gamma / 2}$ for any $\gamma \in(0,1)$, $\mathbf{T}$ is compact. The essential difficulty here is the continuity of the map $\mathbf{T}$. There are many ways to modify $\mathbf{T}$ to a smooth operator $\mathbf{T}^{\varepsilon}$ and show the following:

1. $\quad \mathbf{T}^{\varepsilon}$ admits a fixed point $u^{\varepsilon}$ in $\mathbf{X}(\kappa)$;

2. the family $\left\{u^{\varepsilon}\right\}$ is compact, so along a sequence it converges uniformly to a limit $u$;

3. the family $\left\{\tau^{\varepsilon}\right\}$, where $\tau^{\varepsilon}$ is the arriving time for the domain expansion problem with $v=$ $V\left(u^{\varepsilon}\right)$, is uniformly Lipschitz so along a sequence it converges to a Lipschitz function, say $\hat{\tau}$;

4. the family $\left\{\chi_{\mathrm{D}^{\varepsilon}}\right\}$, where $\mathrm{D}^{\varepsilon}$ is the solution to (1.1) with $v=V \circ u^{\varepsilon}$, converges along a sequence to a limit, $\chi_{\hat{\mathrm{D}}}=1-H(\hat{\tau}(x)-t)$, a.e. in space-time measure, since

$$
\chi_{D^{\varepsilon}(t)}(x)=1-H\left(\tau^{\varepsilon}(x)-t\right) .
$$

The intrinsic question still persists: Is $\{\hat{\mathrm{D}}(t)\}$ the solution $\{\mathrm{D}(t)\}$ to the domain expansion problem with speed $v=V \circ u$ ? Equivalently, is $\hat{\tau}$ the viscosity solution $\tau$ to $v(x, \tau)|\nabla \tau(x)|=1$ ?

It is easy to show that $\tau \leqslant \hat{\tau}$ (i.e. $\hat{\mathrm{D}}(t) \subset \mathrm{D}(t)$ ), but in general it is not clear if $\hat{\tau} \equiv \tau$ is true. If one interprets $\hat{\tau}$ as a weak solution to $v(\cdot, \hat{\tau}(\cdot))|\nabla \hat{\tau}(\cdot)|=1$ then one can call $(u, \hat{\tau})$ a weak solution to the problem and consider the problem as solved. But here we are using the formulation (1.1), not a solution derived from any weak solution of the Hamilton-Jacobi equation for $\phi$ or for $\tau$.

To show $\hat{\tau} \equiv \tau$, we investigate the continuous dependence of domain expansion. We found that there is a continuous dependence provided that there exists a modulo of continuity $\omega$ such that

$$
\int_{0}^{1} \frac{d h}{\omega(h)}=\infty, \quad|v(x, t)-v(y, t)| \leqslant \omega(|x-y|), \quad \forall x, y \in \mathbb{R}^{n}, t \geqslant 0 .
$$

Although $v=V \circ u$ may not satisfy this property, it does satisfy, as shown in [34], that

$$
|\nabla w(x, t)| \leqslant K(1+|\ln \operatorname{dist}(x, \Gamma(t))|), \quad \forall x \in \mathbb{R}^{n}, t \geqslant 0
$$

where $\operatorname{dist}(A, B)$ stands for the distance between sets $A$ and $B$. Such a property essentially gives us a modulo of continuity of the form $\omega(h)=K h[1+|\ln h|]$, thus enabling us to show that $\hat{\tau} \equiv \tau$ and completes the existence proof of a global solution to the free boundary problem (1.1), (1.4), (1.5). 
The plan of this paper is as follows. In $\S 2$, we provide precise definition of the domain expansion problem, together with a few easy to derive properties of the solution and a few illustrative examples. In $\S 3$, we show that every boundary characteristic trajectory has a Hölder continuous direction field. In $\$$, we prove a clearing lemma, i.e. the inclusion property (1.3). We establish our main result (1.2) in $\S 5$, together with a global estimate when the initial set $\mathrm{D}_{0}$ satisfies the interior cone condition. In $\S 6$ we study the stability issue; in particular, we establish stability under the condition (1.6) or (1.7). In $\S 7$ we study regularity of the singular layer potential $w$ under the assumption (1.2). Finally, in $\S 8$, we show that the free boundary problem for solidification admits a global in time solution.

In the sequel, we denote by $C(A ; B)$ the set of continuous functions from $A$ to $B$ and by $\operatorname{dist}(A, B)$ the distance between sets $A$ and $B$. Also, $\mathbb{S}^{n-1}$ is the unit sphere $\left\{x \in \mathbb{R}^{n}|| x \mid=1\right\}$ in $\mathbb{R}^{n}$.

\section{Reachable set, arriving time, and boundary characteristics}

In this section, we assume that

$$
\left\{\begin{array}{l}
v \in C\left(\mathbb{R}^{n} \times[0, \infty) ;[1, c]\right), \quad c \in[1, \infty), \\
\mathrm{D}_{0} \text { is a non-empty bounded closed set. }
\end{array}\right.
$$

We define

$$
\begin{aligned}
\mathrm{C}(t) & :=\left\{\mathbf{x} \in C\left([0, t] ; \mathbb{R}^{n}\right):\left|\mathbf{x}\left(t_{2}\right)-\mathbf{x}\left(t_{1}\right)\right| \leqslant \int_{t_{1}}^{t_{2}} v(\mathbf{x}(s), s) d s, \quad \forall 0 \leqslant t_{1} \leqslant t_{2} \leqslant t\right\}, \\
\mathrm{D}(t) & :=\left\{\mathbf{x}(t) \mid \mathbf{x} \in \mathrm{C}(t), \mathbf{x}(0) \in \mathrm{D}_{0}\right\}, \\
\Gamma(t) & :=\partial D(t), \\
\mathcal{C}_{b}(t) & :=\{\mathbf{x} \in \mathrm{C}(t) \mid \mathbf{x}(s) \in \Gamma(s) \forall s \in[0, t]\}, \\
\tau(x) & :=\inf \{t \geqslant 0 \mid x \in \mathrm{D}(t)\}, \\
\mathrm{Q} & :=\{(x, t) \mid t \geqslant 0, x \in \mathrm{D}(t)\}=\bigcup_{t \geqslant 0} \mathrm{D}(t) \times\{t\} .
\end{aligned}
$$

Definition 2.1 The solution of domain expansion problem with speed $v$ starting from $\mathrm{D}_{0}$ is $Q$. The reachable set at time $t \geqslant 0$ is $\mathrm{D}(t)$ and the (moving) front at time $t$ is $\Gamma(t)$.

The arriving time from $\mathrm{D}_{0}$ to $x \in \mathbb{R}^{n}$ is $\tau(x)$.

A boundary characteristic trajectory is $\mathbf{x}([0, t]):=\{\mathbf{x}(s) \mid s \in[0, t]\}$ where $t>0$ and $\mathbf{x} \in \mathrm{C}_{b}(t)$. A boundary characteristic curve is $\{(\mathbf{x}(s), s) \mid s \in[0, t]\}$ where $t>0$ and $\mathbf{x} \in \mathrm{C}_{b}(t)$.

To help the readers understand our definition, we provide the following lemma. It shows that $\Gamma(t)$ is the $t$-level set of $\tau$, the lateral boundary of Q consists of boundary characteristic curves, and the domain expands with speed $v$.

Lemma 2.1 Assume (2.1).

1. $\mathrm{D}(0)=\mathrm{D}_{0}$ and for each $t>0, \mathrm{D}(t)$ is bounded and closed. In addition,

$$
\begin{aligned}
& \mathrm{D}(s) \subset \mathrm{D}(t), \quad c|t-s| \geqslant \operatorname{dist}(\mathrm{D}(s), x) \geqslant|t-s|, \quad \forall t>s \geqslant 0, x \in \Gamma(t), \\
& \mathrm{D}_{0}=\left\{x \in \mathbb{R}^{n} \mid \tau(x)=0\right\}, \quad \Gamma(t)=\left\{x \in \mathbb{R}^{n} \mid \tau(x)=t\right\}, \quad \forall t>0, \\
& |\tau(x)-\tau(y)| \leqslant|x-y|, \quad \forall x, y \in \mathbb{R}^{2} .
\end{aligned}
$$


2. Regarding $Q$ as a space-time domain,

$$
\begin{aligned}
\partial \mathrm{Q} & =\left(\mathrm{D}_{0} \times\{0\}\right) \cup \partial_{p} \mathrm{Q}, \\
\partial_{p} \mathrm{Q} & :=\bigcup_{t \geqslant 0}(\Gamma(t) \times\{t\})=\bigcup_{t \geqslant 0, \mathbf{x} \in \mathbb{C}_{b}(t)}\{(\mathbf{x}(s), s) \mid s \in[0, t]\} .
\end{aligned}
$$

3. Boundary characteristic curves have speed $v$; that is, if $\mathbf{x} \in \mathrm{C}_{b}(T)$, then

$$
\lim _{[0, T] \ni t_{2}, t_{1} \rightarrow t}\left|\frac{\mathbf{x}\left(t_{2}\right)-\mathbf{x}\left(t_{1}\right)}{t_{2}-t_{1}}\right|=v(\mathbf{x}(t), t), \quad \forall t \in[0, T] .
$$

4. The fronts expand with speed $v$ in the following sense: for every $t>0$,

$$
\lim _{s \nearrow t} \frac{\operatorname{dist}(x, \mathrm{D}(s))}{t-s}=v(x, t) \quad \Longleftrightarrow \quad x \in \Gamma(t) .
$$

REMARK 2.1 The equivalence (2.2) can be indeed used as a definition of weak solutions:

A collection of closed sets $\{\mathrm{D}(t)\}_{t \geqslant 0}$ is called expanding with speed $v$ if $\mathrm{D}(s) \subset \mathrm{D}(t)$ for all $0 \leqslant s<t$ and (2.2) holds with $\Gamma(t):=\partial \mathrm{D}(t)$.

However, we shall not elaborate on this topic.

Proof. (1) Since $v \geqslant 1$, by definition, $\mathrm{D}(0)=D_{0}$. For each $t>0$, we show that $\mathrm{D}(t)$ is closed.

Let $x \in \Gamma(t):=\partial \mathrm{D}(t)$. There exists $\left\{x_{i}\right\}_{i=1}^{\infty} \in \mathrm{D}(t)$ such that $x=\lim _{i \rightarrow \infty} x_{i}$. By the definition of $\mathrm{D}(t)$, for each $i \in \mathbb{N}$, there exists $\mathbf{x}_{i} \in \mathrm{C}(t)$ such that $\mathbf{x}_{i}(0) \in \mathrm{D}_{0}$ and $\mathbf{x}_{i}(t)=x_{i}$. Since $v \leqslant c$, the family $\left\{\mathbf{x}_{i}\right\}_{i=1}^{\infty}$ is uniformly bounded and equicontinuous in $C\left([0, T] ; \mathbb{R}^{2}\right)$. There exist $\mathbf{x} \in C\left([0, t] ; \mathbb{R}^{2}\right)$ and a subsequence, which we still denote by $\left\{\mathbf{x}_{i}\right\}$, such that $\lim _{i \rightarrow \infty} \mathbf{x}_{i}=\mathbf{x}$ uniformly on $[0, t]$. Consequently, $x=\lim _{j \rightarrow \infty} \mathbf{x}_{j}(t)=\mathbf{x}(t)$ and $\mathbf{x}(0)=\lim _{i \rightarrow \infty} \mathbf{x}_{i}(0) \in \mathrm{D}_{0}$. Moreover, for any $t \geqslant t_{2} \geqslant t_{1} \geqslant 0$,

$$
\left|\mathbf{x}\left(t_{2}\right)-\mathbf{x}\left(t_{1}\right)\right|=\lim _{i \rightarrow \infty}\left|\mathbf{x}_{i}\left(t_{2}\right)-\mathbf{x}_{i}\left(t_{1}\right)\right| \leqslant \lim _{i \rightarrow \infty} \int_{t_{1}}^{t_{2}} v\left(\mathbf{x}_{i}(s), s\right) d s=\int_{t_{1}}^{t_{2}} v(\mathbf{x}(s), s) d s .
$$

Hence, $\mathbf{x} \in \mathbb{C}(t)$. As $\mathbf{x}(0) \in \mathrm{D}_{0}, x=\mathbf{x}(t) \in \mathrm{D}(t)$. Thus, $\mathrm{D}(t)$ is closed.

Let $0 \leqslant s<t$ and $x \in \Gamma(t)$. We want to show that $|t-s| \leqslant \operatorname{dist}(\mathrm{D}(s), x) \leqslant c|t-s|$.

Let $y \in \mathrm{D}(s)$. There exists $\mathbf{y} \in \mathrm{C}(s)$ such that $\mathbf{y}(0) \in \mathrm{D}_{0}$ and $\mathbf{y}(s)=y$. For every $p \in B(\mathbf{0}, 1)$, extend the definition domain of $\mathbf{y}$ from $[0, s]$ to $[0, \infty)$ by $\mathbf{y}(\varsigma)=y+(\varsigma-s) p$ for $\varsigma>s$. Then $\mathbf{y} \in \mathrm{C}(t)$ so that $y+(t-s) p \in \mathrm{D}(t)$. Consequently, $B(y,(t-s)) \subset \mathrm{D}(t)$. Thus, $\mathrm{D}(s) \subset \mathrm{D}(t)$ and $\operatorname{dist}(\mathrm{D}(s), x) \geqslant t-s$.

On the other-hand, let $\mathbf{x} \in \mathcal{C}(t)$ satisfy $\mathbf{x}(0) \in \mathrm{D}_{0}$ and $\mathbf{x}(t)=x$. Since $\left.\mathbf{x}\right|_{[0, s]}$, the restriction of $\mathbf{x}$ on $[0, s]$, is in $\mathrm{C}(s)$ we see that $\mathbf{x}(s) \in \mathrm{D}(s)$. Hence,

$$
|x-\mathbf{x}(s)|=|\mathbf{x}(t)-\mathbf{x}(s)| \leqslant(t-s) c
$$

and

$$
\operatorname{dist}(\mathrm{D}(s), x) \leqslant \operatorname{dist}(\mathbf{x}(s), x) \leqslant(t-s) c .
$$

From the definition of $\tau$ and the distance relation it is easy to derive that $\mathrm{D}_{0}=\{x \mid \tau(x)=0\}$ and $\Gamma(t)=\{x \mid \tau(x)=t\}$ for every $t>0$. Now let $x, y \in \mathbb{R}^{n}$. Set $t=\tau(x)$ and $s=\tau(y)$. 
Suppose $t>s$. Then $x \in \Gamma(t)$ and $y \in \mathrm{D}(s)$. It follows that $|x-y| \geqslant \operatorname{dist}(\Gamma(t), \mathrm{D}(s)) \geqslant|t-s|=$ $|\tau(x)-\tau(y)|$. Similarly, we can consider the case $t \leqslant s$. Hence $|\tau(x)-\tau(y)| \leqslant|x-y|$ for all $x, y \in \mathbb{R}^{n}$.

(2) Next we study $\partial \mathrm{Q}$. Since $\operatorname{dist}(\mathrm{D}(0), \Gamma(t)) \geqslant t$, we have $\partial \mathrm{Q} \cap\{t=0\}=\mathrm{D}_{0} \times\{0\}$.

Next we consider the lateral boundary of Q. Let $t>0$ and $(x, t) \in \partial \mathrm{Q}$. Note that $\tau(x)<t$ is impossible since it would imply, setting $\delta=[t-\tau(x)] / 3$ and $\varepsilon=\delta$, that $\tau(y) \leqslant \tau(x)+|x-y|<$ $t-\delta$ for all $y \in B(x, \varepsilon)$ so that $B(x, \varepsilon) \in \mathrm{D}(t-\delta)$ and $B(x, \varepsilon) \times[t-\delta, \infty) \in \mathrm{Q}$, contradicting $(x, t) \in \partial \mathrm{Q}$. Similarly $\tau(x)>t$ is impossible since it would imply $B(x, \varepsilon) \cap \mathrm{D}(t+\delta)=\emptyset$ for $\delta=(\tau(x)-t) / 3$ and $\varepsilon=\delta$, so that $(B(x, \varepsilon) \times[0, t+\delta]) \cap \mathrm{Q}=\emptyset$, contracting $(x, t) \in \partial \mathrm{Q}$. Thus, $\tau(x)=t$. Consequently, $x \in \Gamma(\tau(x))=\Gamma(t)$.

In conclusion, $\partial \mathrm{Q}=\left(\mathrm{D}_{0} \times\{0\}\right) \cup \partial_{p} Q$ where $\partial_{p} \mathrm{Q}:=\cup_{t>0}(\Gamma(t) \times\{t\})$.

Now we show that $\partial_{p} \mathrm{Q}$ consists of boundary characteristic curves. It suffices to show that for each $T>0$ and $x \in \Gamma(T)$, there exists $\mathbf{x} \in \mathrm{C}_{b}(T)$ such that $x=\mathbf{x}(T)$. The proof is straightforward when $v$ is Lipschitz continuous in the space variable but quite delicate when $v$ is merely continuous. (a) First we consider the case that $v$ is Lipschitz continuous.

Let $\mathbf{x} \in \mathrm{C}(T)$ be such that $\mathbf{x}(0) \in \mathrm{D}_{0}$ and $\mathbf{x}(T)=x$. We claim that $\mathbf{x} \in \mathrm{C}_{b}(T)$, i.e $\mathbf{x}(s) \in \Gamma(s)$ for all $s \in[0, T]$. For this, we use a contradiction argument. Suppose $\mathbf{x} \notin \mathfrak{C}_{b}(T)$. Then for some $s_{0} \in[0, T), \mathbf{x}\left(s_{0}\right)$ is an interior point of $\mathrm{D}\left(s_{0}\right)$, so there exists $\varepsilon>0$ such that $B\left(\mathbf{x}\left(s_{0}\right), \varepsilon\right) \subset \mathrm{D}\left(s_{0}\right)$.

Let $\left\{x_{i}\right\}_{i=1}^{\infty}$ be a sequence such that $x_{i} \notin \mathrm{D}(T)$ but $\left|x-x_{i}\right|<1 / i$ for all $i \in \mathbb{N}$. Since $\mathbf{x}$ is Lipschitz continuous, $\mathbf{x}$ is differentiable almost everywhere. Now for every $i \in \mathbb{N}$, consider integral equation

$$
\mathbf{y}_{i}(t)=x_{i}+\int_{T}^{t} v\left(\mathbf{y}_{i}(s), s\right) \frac{d \mathbf{x}(s)}{v(\mathbf{x}(s), s)}, \quad \forall t \in\left[s_{0}, T\right] .
$$

Since $v(\cdot, \cdot)$ is Lipschitz continuous and bounded, there is a unique solution. By taking a subsequence if necessary, there exists $\mathbf{y} \in C\left(\left[s_{0}, T\right]\right)$ such that $\lim _{i \rightarrow \infty} \mathbf{y}_{i}=\mathbf{y}$ uniformly on $\left[s_{0}, T\right]$. In addition,

$$
\mathbf{y}(t)=x+\int_{T}^{t} v(\mathbf{y}(s), s) \frac{d \mathbf{x}(s)}{v(\mathbf{x}(s), s)}, \quad \forall t \in\left[s_{0}, T\right] .
$$

Since $v$ is Lipschitz continuous, the solution is unique. Hence, $\mathbf{y}(t)=\mathbf{x}(t)$ for all $t \in\left[s_{0}, T\right]$. Consequently, for some $j$ large enough, $\mathbf{y}_{j}\left(s_{0}\right) \in B\left(\mathbf{x}\left(s_{0}\right), \varepsilon\right) \subset \mathrm{D}\left(s_{0}\right)$. Let $\mathbf{z} \in \mathbb{C}\left(s_{0}\right)$ be such that $\mathbf{z}(0) \in \mathrm{D}_{0}$ and $\mathbf{z}\left(s_{0}\right)=\mathbf{y}_{j}\left(s_{0}\right)$. Extend $\mathbf{y}_{j}$ from $\left[s_{0}, T\right]$ to $[0, T]$ by $\mathbf{y}_{j}(t)=\mathbf{z}(t)$ for $t \in\left[0, s_{0}\right)$. Since $|\dot{\mathbf{x}}(t)| \leqslant v(\mathbf{x}(t), t)$ for all most every $t \in\left[s_{0}, T\right]$, one can check that $\mathbf{y}_{j} \in \mathrm{C}(T)$, so $\mathbf{y}_{j}(T)=$ $x_{j} \in \mathrm{D}(T)$. But this is impossible. Thus, $\mathbf{x} \in \mathrm{C}_{b}(T)$.

(b) Now we are ready to consider the general case that $v$ is merely continuous.

By mollification, there exist $\left\{v_{i}\right\}_{i=1}^{\infty}$ of $C^{\infty}\left(\mathbb{R}^{n} \times[0, \infty),[1, c+2]\right)$ functions such that

$$
v(x, t)+2^{-i}<v_{i}(x, t)<v(x, t)+2^{1-i}, \quad \forall(x, t) \in B(0, R) \times[0, T], j \in \mathbb{N}
$$

where $R$ is a large constant such that $\mathrm{D}_{0} \subset B(0, R-2-[2+c] T)$.

Let $\mathrm{Q}_{j}=\cup_{t \geqslant 0}\left(\mathrm{D}_{j}(t) \times\{t\}\right)$ be the solution to the domain expansion with speed $v_{j}$ starting from $\mathrm{D}_{0}$ :

$$
\begin{aligned}
& \mathrm{D}_{j}(t)=\left\{\mathbf{x}(t) \mid \mathbf{x}(0) \in \mathrm{D}_{0}, \mathbf{x} \in \mathcal{C}_{j}(t)\right\}, \\
& \mathcal{C}_{j}(t)=\left\{\mathbf{x} \in C([0, t])|| \mathbf{x}\left(t_{2}\right)-\mathbf{x}\left(t_{1}\right) \mid \leqslant \int_{t_{1}}^{t_{2}} v_{j}(\mathbf{x}(s), s) d s, \quad \forall 0 \leqslant t_{1} \leqslant t_{2} \leqslant t\right\} .
\end{aligned}
$$


For every $\mathbf{x} \in \mathrm{C}(t)$ and $p \in B(0,1)$, the function $s \in[0, t] \rightarrow \mathbf{x}(s)+2^{-j} t p$ is in $\mathrm{C}_{j}(t)$ so $\mathbf{x}(t)+2^{-j} t p \subset \mathrm{D}_{j}(t)$. Thus, $\operatorname{dist}\left(\mathrm{D}(t), \Gamma_{j}(t)\right) \geqslant 2^{-j} t$.

Now let $x \in \Gamma(T)$ be an arbitrary point. For each $j$, let $t_{j} \in[0, T)$ be such that $x \in \Gamma_{j}\left(t_{j}\right)$. Since $v_{j+1}<v_{j}$, we see that $\mathrm{D}_{j+1}(t) \subset \mathrm{D}_{j}(t)$ for all $t$ so that $\left\{t_{j}\right\}$ is an increasing sequence, bounded from above by $T$. Denote $T^{*}=\lim _{j \rightarrow \infty} t_{j}$.

Let $\mathbf{x}_{j} \in \mathrm{C}_{j}\left(t_{j}\right)$ be such that $\mathbf{x}_{j}(0) \in \mathrm{D}_{0}$ and $\mathbf{x}_{j}\left(t_{j}\right)=x$. Since $v_{j}$ is smooth, by (a), we know that $\mathbf{x}_{j}(t) \in \Gamma_{j}(t)$ for all $t \in\left[0, t_{j}\right]$ so that

$$
\operatorname{dist}\left(\mathbf{x}_{j}(t), \mathrm{D}(t)\right) \geqslant 2^{-j} t, \quad \forall t \in\left[0, t_{j}\right] .
$$

Extend $\mathbf{x}_{j}$ from $\left[0, t_{j}\right]$ to $[0, T]$ by $\mathbf{x}_{j}(t)=x$ for all $t \in\left[t_{j}, T\right]$. Then $\left\{\mathbf{x}_{j}\right\}$ is a uniformly bounded and equicontinuous family in $C\left([0, T] ; \mathbb{R}^{n}\right)$, so there exists a subsequence, which we still denote by $\left\{\mathbf{x}_{j}\right\}$, such that for some $\mathbf{x} \in C\left([0, T] ; \mathbb{R}^{n}\right)$,

$$
\lim _{j \rightarrow \infty}\left\|\mathbf{x}_{j}-\mathbf{x}\right\|_{C\left([0, T] ; \mathbb{R}^{n}\right)}=0 .
$$

Following same argument as that used in step (i), we can show that $\mathbf{x} \in \mathrm{C}(T)$. Hence, $\mathbf{x}(t) \in \mathrm{D}(t)$ for all $t \in[0, T]$. Clearly, $\mathbf{x}\left(T^{*}\right)=\lim _{j \rightarrow \infty} \mathbf{x}_{j}\left(T^{*}\right)=x$. Since $x \in \Gamma(T)$, we must have $T^{*}=T$. Finally, for any $t \in(0, T), t_{j}>t$ for all large enough $j$ so that $\operatorname{dist}\left(\mathbf{x}_{j}(t), \mathrm{D}(t)\right) \geqslant 2^{-j} t$ for all large enough $j$. This implies that $\mathbf{x}(t)=\lim _{j \rightarrow \infty} \mathbf{x}_{j}(t) \in \partial \mathrm{D}(t)$. Thus, $\mathbf{x} \in \mathrm{C}_{b}(T)$.

(3) We now show that boundary characteristic moves with speed $v$, using an argument from [34].

Let $T>0$ and $\mathbf{x} \in \mathrm{C}_{b}(T)$ be arbitrary. Pick arbitrary $t_{1}$ and $t_{2}$ satisfying $0 \leqslant t_{1}<t_{2} \leqslant T$. We define

$$
\mathbf{e}\left(t_{1}, t_{2}\right)=\frac{\mathbf{x}\left(t_{2}\right)-\mathbf{x}\left(t_{1}\right)}{\left|\mathbf{x}\left(t_{2}\right)-\mathbf{x}\left(t_{1}\right)\right|}
$$

Let $\theta:\left[t_{1}, \infty\right) \rightarrow \mathbb{R}$ be a solution to the ordinary differential equation

$$
\dot{\theta}(t)=v\left(\mathbf{x}\left(t_{1}\right)+\theta(t) \mathbf{e}\left(t_{1}, t_{2}\right), t\right), \quad \forall t \in\left[t_{1}, \infty\right), \quad \theta\left(t_{1}\right)=0 .
$$

Define

$$
\mathbf{y}(t)= \begin{cases}\mathbf{x}(t) & \text { if } t \in\left[0, t_{1}\right], \\ \mathbf{x}\left(t_{1}\right)+\theta(t) \mathbf{e}\left(t_{1}, t_{2}\right) & \text { if } t \in\left[t_{1}, T\right] .\end{cases}
$$

Then $\mathbf{y} \in \mathrm{C}(T)$ since

$$
\dot{\theta}(t)=v(\mathbf{y}(t), t), \quad \dot{\mathbf{y}}=v(\mathbf{y}(t), t) \mathbf{e}\left(t_{1}, t_{2}\right), \quad \forall t \geqslant t_{1} .
$$

Note that $\dot{\theta} \geqslant 1$ in $\left[t_{1}, \infty\right)$. There exists $t^{*}>t_{1}$ such that $\theta\left(t^{*}\right)=\left|\mathbf{x}\left(t_{2}\right)-\mathbf{x}\left(t_{1}\right)\right|$. Then $\mathbf{y}\left(t^{*}\right)=$ $\mathbf{x}\left(t_{2}\right)$. Since $\mathbf{x}\left(t_{2}\right) \in \Gamma\left(t_{2}\right)$, we must have $t^{*} \geqslant t_{2}$. It then follows that

$$
\left|\mathbf{x}\left(x_{2}\right)-\mathbf{x}\left(t_{1}\right)\right|=\theta\left(t^{*}\right) \geqslant \theta\left(t_{2}\right)=\int_{t_{1}}^{t_{2}} v(\mathbf{y}(t), t) d t=\int_{t_{1}}^{t_{2}}|\dot{\mathbf{y}}(t)| d t .
$$

On the other-hand,

$$
\left|\mathbf{x}\left(x_{2}\right)-\mathbf{x}\left(t_{1}\right)\right|=\mathbf{e}\left(t_{1}, t_{2}\right) \cdot \int_{t_{1}}^{t_{2}} \dot{\mathbf{x}}(t) d t \leqslant \int_{t_{1}}^{t_{2}}|\dot{\mathbf{x}}(t)| d t \leqslant \int_{t_{1}}^{t_{2}} v(\mathbf{x}(t), t) d t .
$$


Hence, we have

$$
\frac{1}{t_{2}-t_{1}} \int_{t_{1}}^{t_{2}} v(\mathbf{y}(t), t) d t \leqslant\left|\frac{\mathbf{x}\left(t_{2}\right)-\mathbf{x}\left(t_{1}\right)}{t_{2}-t_{1}}\right| \leqslant \frac{1}{t_{2}-t_{2}} \int_{t_{1}}^{t_{2}} v(\mathbf{x}(t), t) d t .
$$

Now fix any $t \in[0, T]$. Sending $t_{2}, t_{1} \rightarrow t$ we obtain

$$
\lim _{t_{2}, t_{1} \rightarrow t}\left|\frac{\mathbf{x}\left(t_{2}\right)-\mathbf{x}\left(t_{1}\right)}{t_{2}-t_{1}}\right|=v(\mathbf{x}(t), t) \quad \forall t \in[0, T] .
$$

(4) Finally we prove the equivalence (2.2). Let's fix $t>0$.

“ $\Rightarrow$ ": Suppose $x$ satisfies

$$
\lim _{s \nearrow t} \operatorname{dist}(x, \Gamma(s)) /(t-s)=v(x, t)
$$

Then

$$
\operatorname{dist}(x, \mathrm{D}(t)) \leqslant \lim _{s \nearrow t} \operatorname{dist}(x, \mathrm{D}(s))=0
$$

so that $x \in \mathrm{D}(t)$. It is then easy to see that $x \in \Gamma(t)$.

" $\Leftarrow$ ": Suppose $x \in \Gamma(t)$. Then by using an $\mathbf{x} \in \mathrm{C}(t)$ with $\mathbf{x}(t)=x$ and $\mathbf{x}(0) \in \mathrm{D}_{0}$ we see that

$$
\limsup _{s \nearrow t} \operatorname{dist}(x, \Gamma(s)) /(t-s) \leqslant \limsup _{s \nearrow t}|\mathbf{x}(t)-\mathbf{x}(s)| /(t-s) \leqslant v(x, t) .
$$

Let

$$
v_{*}=\liminf _{s \nearrow t} \operatorname{dist}(x, \mathrm{D}(s)) /(t-s) .
$$

If $v_{*}<v(x, t)-3 \varepsilon$ for some $\varepsilon>0$, then $v>v_{*}+2 \varepsilon$ in $B(x, c \delta) \times[t-\delta, t]$ for some $\delta>0$. Pick $s \in[t-\delta, t)$ and $y \in \mathrm{D}(s)$ such that

$$
|x-y|=\operatorname{dist}(x, \mathrm{D}(s))<\left(v^{*}+\varepsilon\right)(t-s)<c \delta .
$$

Consider the curve

$$
\mathbf{z}(\varsigma):=y+(\varsigma-s)\left(v_{*}+2 \varepsilon\right)(x-y) /|x-y| .
$$

Since

$$
(t-s)\left(v_{*}+2 \varepsilon\right) /|x-y|>\left(v_{*}+2 \varepsilon\right) /\left(v_{*}+\varepsilon\right)>1,
$$

there is $t^{*} \in(s, t)$ such that $\mathbf{z}\left(t^{*}\right)=x$. Also $|\dot{\mathbf{z}}(\varsigma)|=v^{*}+2 \varepsilon \leqslant v(\mathbf{z}, \varsigma)$ for all $\varsigma \in\left[s, t^{*}\right]$ so that $x=\mathbf{z}\left(t^{*}\right) \in \mathrm{D}\left(t^{*}\right)$, contradicting $x \in \Gamma(t)$. Thus, $v_{*}=v(x, t)$ and (2.2) holds. This completes the proof of the Lemma.

We end this section with a few examples. The first three are one dimensional examples illustrating the complication that may arise when $v$ is not Lipschitz continuous in the space variable. The last three are two dimensional examples, one illustrating that boundary characteristic trajectories are perpendicular to the moving fronts $\{\Gamma(t)\}$, another one illustrating that $\Gamma(t)$ could form countably many "islands", and the last one calculating a few quantities that will demonstrate the optimality of our estimates in subsequent sections. We remark here that since the expanding speed is finite, the unboundedness of the initial shape in some of our examples won't be an issue. 
Example 1 Let $\Omega_{0}=(-1,0)$ and $D_{0}=[-1,0]$. Consider $v(x, t)=1+2 \sqrt{\max \{x-t, 0\}}$. Define $\Omega(t)=\left\{\mathbf{x}(t) \mid \mathbf{x}(0) \in \Omega_{0}, \mathbf{x} \in \mathbb{C}(t)\right\}$. Then one sees that

$$
\Omega(t)=(-1-t, t), \quad \mathrm{D}(t)=\left[-1-t, t+t^{2}\right] \quad \forall t \geqslant 0 .
$$

Clearly, $\mathrm{D}(t) \neq \overline{\Omega(t)}$ for any $t>0$.

EXAMPLE 2 Let $\mathrm{D}_{0}=(-\infty, 0], \Omega_{0}=(-\infty, 0)$ and

$$
v(x, t)=1+2 \sqrt{\max \{t-x, 0\}} .
$$

It is easy to see that $\mathrm{D}(t)=\Omega(t)=(-\infty, t]$ for all $t>0$. There is only one boundary characteristic curve $\{(t, t) \mid t \in[0, \infty)\}$. Nevertheless, given any boundary point $(T, T)(T>0)$, there are infinitely many curves in $\mathrm{C}(T)$ that starts in $\mathrm{D}_{0}$ and ends at the boundary boundary point $(T, T)$.

Thus, when $v$ is not Lipschitz continuous, it is better to study evolution of closed sets. Also a curve described by a function in $\mathrm{C}(T)$ that starts and ends on $\partial \mathrm{Q}$ is not necessarily a boundary characteristic curve; that is, a particle starting from the interior of a region with speed no more than $v$ can in finite time move to the frontier of the region which expands with speed $v$.

The following one space dimensional example illustrates the instability of the domain expansion problem when $v$ is only Hölder continuous.

ExAmple 3 Let $\mathrm{D}_{0}=(-\infty, 0], v(x, t)=1+2 \sqrt{\max \{x-t, 0\}}$ for all $(x, t) \in \mathbb{R} \times[0, \infty)$. The solution to domain expansion with speed $v$ starting from $\mathrm{D}_{0}$ is $\mathrm{D}(t)=\left(-\infty, t+t^{2}\right]$ for all $t \geqslant 0$. The arriving time $\tau(x)$ is obtained by solving $\tau+\tau^{2}=x$ so

$$
\tau(x)=\sqrt{x+1 / 4}-1 / 2, \quad \forall x \geqslant 0 .
$$

Let $\mathrm{D}_{0 i}=(-\infty,-1 / i]$ for every $i \in \mathbb{N}$. The solution of domain expansion with speed $v$ starting from $\mathrm{D}_{0 i}$ is $\mathrm{D}_{i}(t)=(-\infty, t-1 / i]$ for all $t \in[0, \infty)$ and all $i \in \mathbb{N}$. The arriving time is given by $\tau_{i}(x):=x+1 / i$ for every $x \geqslant-1 / i$.

Let

$$
v_{j}(x, t)=1+2 \sqrt{1 / j^{2}+\max \{x-t, 0\}}-2 / j \text { for all } j \in \mathbb{N} .
$$

It is easy to see that each $v_{j}$ is Lipschitz continuous and the solution with speed $v_{j}$ starting from $\mathrm{D}_{0}$ is given by $\mathrm{D}_{0}(t)=(-\infty, t]$ so that $\tau_{j}(x)=x$ for every $x \geqslant 0$.

Clearly, $\hat{\tau}(x):=\lim _{j \rightarrow \infty} \tau_{j}(x)=x$ for each $x \geqslant 0$. The function $\hat{\tau}$ is a classical solution to the equation

$$
v(x, \hat{\tau}(x))|\nabla \hat{\tau}(x)|=1 .
$$

However, according to the Lax formula (1.1), the arriving time for the unique viscosity solution is given by

$$
\tau(x)=\sqrt{x+1 / 4}-1 / 2,
$$

$\operatorname{not} \hat{\tau}$.

In the smooth case, the velocity vector of every boundary characteristic trajectory is the outward normal vector of the evolution domain at the front. Hence, the family of boundary characteristic trajectories and the family of fronts (level sets of $\tau$ ) are orthogonal to each other. 


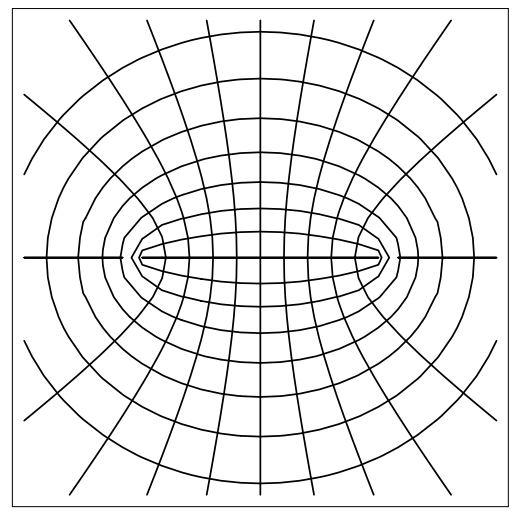

(a)

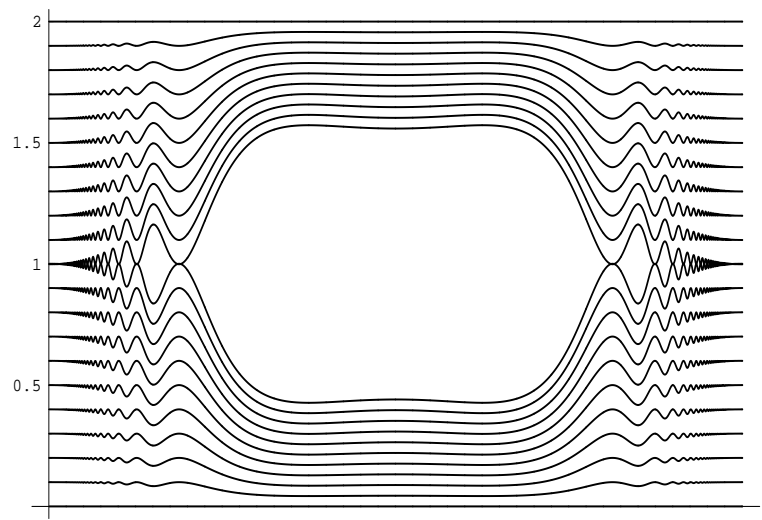

(b)

FIG. 1. (a) Boundary characteristic trajectories (hyperbolas) are orthogonal to the moving fronts (ellipses).

(b) A numerical example $\left(v(x, y, t)=1-0.8\left(\sin x \sin \frac{1}{0.01+\sin ^{2} x}\right)^{2}\right)$ illustrates the formation of countably many "islands".

EXAMPLE 4 Assume that $n=2, D_{0}=\Gamma_{0}=[-1,1] \times\{0\}$, and

$$
v(t, x, y)=\sqrt[4]{\left[1-x^{2}-y^{2}\right]^{2}+4 y^{2}} .
$$

Using complex variables, one can verify that

$$
\Gamma(t)=\left\{(x, y) \in \mathbb{R}^{2} \mid \frac{x^{2}}{\cosh ^{2} t}+\frac{y^{2}}{\sinh ^{2} t}=1\right\} \quad \forall t>0 .
$$

Hence, each $\Gamma(t)$ is an ellipse. All boundary characteristic trajectories can be parameterized by a parameter $\theta \in[0,2 \pi)$, and is given by

$$
\mathbf{x}(t)=(\cos \theta \cosh t, \sin \theta \sinh t), \quad t \in[0, \infty)
$$

where $(\cos \theta, 0) \in \mathrm{D}_{0}$ is the starting point. All these boundary characteristic trajectories form a family of hyperbolas. The hyperbola family of boundary characteristic trajectories and the ellipse family of fronts form an orthogonal net; see Figure 1(a).

The fact that boundary characteristic trajectories are in general perpendicular to moving fronts suggests that the regularity of moving fronts is related to regularity of boundary characteristic trajectories. Hence, to study regularities of moving fronts, it is better to begin with studying regularities of boundary characteristic trajectories, as we shall do in the next section. The central difficulty in transferring the regularity of boundary characteristic trajectories to that of moving fronts is the geometric fact that boundary characteristic trajectories can intersect each other, thereby creating "corners" of the moving fronts and "islands" for the complement of moving domains. The following example illustrates that countably many islands can be formed in finite time from smooth initial fronts.

EXAMPLE 5 Let $n=2, \mathrm{D}_{0}=\mathbb{R} \times((-\infty, 0] \cup[2, \infty))$, and consider $C^{1}$ function on $\mathbb{R}^{2} \times[0, \infty)$

$$
v(x, y, t)=1-\frac{\sin ^{2} x}{2} \sin ^{2} \frac{1}{\sin x} \text { if } \sin x \neq 0, \quad v(x, y, t)=1 \text { if } \sin x=0 .
$$


Set $x_{m, 0}=m \pi$ and $x_{m, k}=m \pi+\arcsin \frac{1}{k \pi}$ for $k \neq 0$. Note that for any integers $k$ and $m$, the boundary characteristic trajectory starting from $\left(x_{m, k}, 0\right)$ and $\left(x_{m, k}, 2\right)$ form a vertical line $\left\{x_{m, k}\right\} \times$ $[0,2]$. One expect (indeed can show by solving a partial differential equation) that there exists a spatially $\pi$-periodic $C^{1}(\mathbb{R} \times[0,1])$ function $\psi(\cdot, \cdot)$ such that for every $t \in[0,1]$,

$$
\Gamma(t)=\{(x, y) \mid x \in \mathbb{R}, \quad y=\psi(x, t) \text { or } y=2-\psi(x, t)\} .
$$

In addition, for every $t \in[0,1]$,

$$
\psi\left(x_{m, k}, t\right)=t, \quad \forall m, k \in \mathbb{Z}, \quad \frac{1}{2} t<\psi(x, t)<t, \quad \forall x \notin \bigcup_{m, k \in \mathbb{Z}}\left\{x_{m, k}\right\} .
$$

Thus, the complement of D(1) contains countably many "islands"; see Figure 1(b).

In the following two-dimensional example, we investigate the regularity of the boundary characteristic trajectories, the comparison between trajectory length and line distance, as well as the regularity of the fronts. One will see that the appearance of the exponents $\alpha / 2$ and $\beta:=\alpha /(2-\alpha)$ is natural.

EXAMPLE 6 Let $n=2$ and $\alpha \in(0,1)$ be fixed. Consider

$$
\hat{\mathrm{D}}_{0}:=\left\{\left.(x, y)|y \in \mathbb{R}, x \leqslant-| y\right|^{1+\alpha / 2}\right\}, \quad v(x, y, t):=\left\{1+\left(1+\frac{\alpha}{2}\right)^{2}|y|^{\alpha}\right\}^{-\frac{1}{2}} .
$$

1. The solution with speed $v$ starting from $\hat{\mathrm{D}}_{0}$ is given by

$$
\hat{\tau}(x, y)=x+|y|^{1+\alpha / 2}, \quad \hat{\Gamma}(t)=\left\{\left.(x, y)|y \in \mathbb{R}, x=t-| y\right|^{1+\alpha / 2}\right\} .
$$

The normal to the fronts are $\left(1,\left(1+\frac{1}{2} \alpha\right) y|y|^{\alpha / 2-1}\right)$ so the system of ordinary differential equations for the boundary characteristic trajectories is

$$
\frac{d(x, y)}{d t}=\frac{\left(1,\left[1+\frac{1}{2} \alpha\right] y|y|^{\alpha / 2-1}\right)}{1+\left(1+\frac{1}{2} \alpha\right)^{2}|y|^{\alpha}} \quad \text { or } \quad \frac{d y}{d x}=\left(1+\frac{1}{2} \alpha\right)|y|^{\alpha / 2} \frac{y}{|y|} .
$$

Denote

$$
\Sigma:=\left\{(x, y)|| y \mid \leqslant\left(\left[1-\frac{1}{4} \alpha^{2}\right] x\right)^{1+\beta}\right\} .
$$

For every $p \in \Sigma$, there is a unique boundary characteristic trajectory reaching $p$; see Figure 2(b). This trajectory starts from the origin and can be expressed as

$$
|y|=\left(1-\frac{1}{4} \alpha^{2}\right)^{1+\beta}\left(\max \left\{x-t_{0}, 0\right\}\right)^{1+\beta}, \quad t_{0} \geqslant 0, \quad \beta=\frac{\alpha}{2-\alpha} .
$$

2. Let $\mathrm{D}_{0}$ be any set satisfying $\mathrm{D}_{0} \subset \hat{\mathrm{D}}_{0}$ and $(0,0) \in \mathrm{D}_{0}$. Then in $\Sigma$ the fronts starting from $\mathrm{D}_{0}$ with speed $v$ are the same as above:

$$
\Gamma(t) \cap \Sigma=\Sigma \cap\left\{\left.(x, y)\left|y \in \mathbb{R}^{n}, x=t-\right| y\right|^{1+\alpha / 2}\right\} .
$$

3. Consider the boundary characteristic trajectory given by (2.5). The time $t$ to arrive the point $(x, y)$ can be calculated by solving

$$
t=x+\left(\left[1-\frac{1}{4} \alpha^{2}\right]\left[x-t_{0}\right]\right)^{1+2 \beta} .
$$


Denote $\mathbf{n}(t)=\dot{\mathbf{x}}(t) /|\dot{\mathbf{x}}(t)|$. We have $\mathbf{n}\left(t_{0}\right)=(1,0)$ and for $t>t_{0}$,

$$
\mathbf{n}(t)=\left(1, C\left[x-t_{0}\right]^{\beta}\right) / \sqrt{1+C^{2}\left[x-t_{0}\right]^{2 \beta}}
$$

with $C=\left(1+\frac{1}{2} \alpha\right)\left(1-\frac{1}{4} \alpha^{2}\right)^{\beta}$. Hence,

$$
\left|\mathbf{n}(t)-\mathbf{n}\left(t_{0}\right)\right|=C\left[x-t_{0}\right]^{\beta}+O\left(\left[x-t_{0}\right]^{2 \beta}\right)=C\left[t-t_{0}\right]^{\beta}+O\left(\left[t-t_{0}\right]^{2 \beta}\right) .
$$

Also, we calculate the trajectory length $\ell$ of $\mathbf{x}\left(\left[t_{0}, t\right]\right)$ and line distance $l$ from $\mathbf{x}\left(t_{0}\right)$ to $\mathbf{x}(t)=$ : $(x, y)$. Writing $C_{1}=\left(1-\frac{1}{4} \alpha^{2}\right)^{1+\beta}$, we have, when $0<t-t_{0} \ll 1$,

$$
\begin{aligned}
\ell-l & =\int_{t_{0}}^{t}|\dot{\mathbf{x}}(s)| d s-\left|\mathbf{x}(t)-\mathbf{x}\left(t_{0}\right)\right| \\
& =\int_{t_{0}}^{x} \sqrt{1+\left[(1+\beta) C_{1} z^{\beta}\right]^{2}} d z-\sqrt{\left[x-t_{0}\right]^{2}+C_{1}^{2}\left[x-t_{0}\right]^{2+2 \beta}} \\
& =\left\{\frac{(1+\beta)^{2}}{2(1+2 \beta)}-\frac{1}{2}\right\} C_{1}\left[x-t_{0}\right]^{1+2 \beta}+O\left(\left[x-t_{0}\right]^{1+4 \beta}\right) \\
& =\frac{\beta^{2}\left[t-t_{0}\right]^{1+2 \beta}}{2(1+2 \beta)}+O\left(\left[t-t_{0}\right]^{1+4 \beta}\right)=\frac{\beta^{2} l^{1+2 \beta}}{2(1+2 \beta)}+O\left(l^{1+4 \beta}\right) .
\end{aligned}
$$

\section{Regularity of boundary characteristic trajectories}

In this section, we study the regularity of boundary characteristic trajectories. For this purpose, we shall assume that $v(x, t)$ is Hölder continuous in $x$ : For some $\alpha \in(0,1]$ and $\kappa>0$,

$$
|v(x, t)-v(y, t)| \leqslant \kappa^{\alpha}|x-y|^{\alpha}, \quad \forall x, y \in \mathbb{R}^{n}, t \in[0, \infty) .
$$

Theorem 2 Assume (2.1) and (3.1). For every $T>0$ and $\mathbf{x} \in \mathcal{C}_{b}(T)$, there exists $\mathbf{n} \in$ $C\left([0, T] ; \mathbb{S}^{n-1}\right)$ such that $\dot{\mathbf{x}}(t)=\mathbf{n}(t) v(\mathbf{x}(t), t)$ for all $t \in[0, T]$. In addition, for every $t_{1}, t_{2} \in[0, T]$,

$$
\left|\mathbf{n}\left(t_{1}\right)-\mathbf{n}\left(t_{2}\right)\right| \leqslant M \kappa^{\beta}\left|t_{2}-t_{1}\right|^{\beta}, \quad \beta:=\frac{\alpha}{2-\alpha}, \quad M:=\frac{15 c^{\frac{\beta}{2}}}{1-2^{-\beta}} .
$$

Proof. We divide the proof into several steps.

1. Using the same notation as in part (3) of the proof of Lemma 2.1, we see from (2.3) and (2.4) that

$$
\int_{t_{1}}^{t_{2}}|\dot{\mathbf{y}}(t)| d t \leqslant\left|\mathbf{x}\left(t_{2}\right)-\mathbf{x}\left(t_{1}\right)\right|=\int_{t_{1}}^{t_{2}} \mathbf{e}\left(t_{1}, t_{2}\right) \cdot \dot{\mathbf{x}}(t) d t
$$

Consequently,

$$
\begin{aligned}
\int_{t_{1}}^{t_{2}}|\dot{\mathbf{x}}(t)|\left|\mathbf{e}\left(t_{1}, t_{2}\right)-\frac{\dot{\mathbf{x}}(t)}{|\dot{\mathbf{x}}(t)|}\right|^{2} d t & =2 \int_{t_{1}}^{t_{1}}|\dot{\mathbf{x}}(t)| d t-2 \int_{t_{1}}^{t_{2}} \mathbf{e}\left(t_{1}, t_{2}\right) \cdot \dot{\mathbf{x}}(t) d t \\
& \leqslant 2 \int_{t_{1}}^{t_{2}}\{|\dot{\mathbf{x}}(t)|-|\dot{\mathbf{y}}(t)|\} d t
\end{aligned}
$$


Now using $\mathbf{x}\left(t_{1}\right)=\mathbf{y}\left(t_{1}\right)$ we find that

$$
\begin{aligned}
\max _{t \in\left[t_{1}, t_{2}\right]}|\mathbf{x}(t)-\mathbf{y}(t)|^{2} & \leqslant\left(\int_{t_{1}}^{t_{2}}|\dot{\mathbf{x}}(t)-\dot{\mathbf{y}}(t)| d t\right)^{2} \leqslant\left(t_{2}-t_{1}\right) \int_{t_{1}}^{t_{2}}|\dot{\mathbf{x}}-\dot{\mathbf{y}}|^{2} d t \\
& =\left(t_{2}-t_{1}\right) \int_{t_{1}}^{t_{2}}\left\{(|\dot{\mathbf{x}}|-|\dot{\mathbf{y}}|)^{2}+|\dot{\mathbf{x}}||\dot{\mathbf{y}}|\left|\frac{\dot{\mathbf{y}}}{|\dot{\mathbf{y}}|}-\frac{\dot{\mathbf{x}}}{|\dot{\mathbf{x}}|}\right|^{2}\right\} d t \\
& \leqslant\left(t_{2}-t_{1}\right)\left(c \int_{t_{1}}^{t_{2}}|| \dot{\mathbf{x}}|-| \dot{\mathbf{y}}|| d t+c \int_{t_{1}}^{t_{2}}|\dot{\mathbf{x}}|\left|\mathbf{e}\left(t_{1}, t_{2}\right)-\frac{\dot{\mathbf{x}}}{|\dot{\mathbf{x}}|}\right|^{2} d t\right)
\end{aligned}
$$

since $|\dot{\mathbf{x}}|=v(\mathbf{x}, t) \leqslant c$ and $|\dot{\mathbf{y}}|=v(\mathbf{y}, t) \leqslant c$. By (3.2) for the second integral, we then obtain

$$
\begin{aligned}
\max _{t \in\left[t_{1}, t_{2}\right]}|\mathbf{x}(t)-\mathbf{y}(t)|^{2} & \leqslant 3 c\left(t_{2}-t_{1}\right) \int_{t_{1}}^{t_{2}}|| \dot{\mathbf{x}}|-| \dot{\mathbf{y}}|| d t \\
& =3 c\left(t_{2}-t_{1}\right) \int_{t_{1}}^{t_{2}}|v(\mathbf{x}(t), t)-v(\mathbf{y}(t), t)| d t \\
& \leqslant 3 c\left(t_{2}-t_{1}\right)^{2} \max _{t_{1} \leqslant t \leqslant t_{2}} \kappa^{\alpha}|\mathbf{x}(t)-\mathbf{y}(t)|^{\alpha} ; \\
\max _{t_{1} \leqslant t \leqslant t_{2}}|\mathbf{x}(t)-\mathbf{y}(t)| & \leqslant\left[3 c\left(t_{2}-t_{1}\right)^{2}\right]^{\frac{1}{2-\alpha}} \kappa^{\beta}
\end{aligned}
$$

where $\beta:=\alpha /(2-\alpha)$. Hence, using (3.2) and the above estimates,

$$
\begin{aligned}
\int_{t_{1}}^{t_{2}}\left|\mathbf{e}\left(t_{1}, t_{2}\right)-\frac{\dot{\mathbf{x}}(t)}{|\dot{\mathbf{x}}(t)|}\right| d t & \leqslant\left(\int_{t_{1}}^{t_{2}} \frac{d t}{|\dot{\mathbf{x}}(t)|} \int_{t_{1}}^{t_{2}}|\dot{\mathbf{x}}(t)|\left|\mathbf{e}\left(t_{1}, t_{2}\right)-\frac{\dot{\mathbf{x}}(t)}{|\dot{\mathbf{x}}(t)|}\right|^{2} d t\right)^{1 / 2} \\
& \leqslant\left(\left(t_{2}-t_{1}\right) \int_{t_{1}}^{t_{2}} 2 \kappa^{\alpha}|\mathbf{x}(t)-\mathbf{y}(t)|^{\alpha} d t\right)^{1 / 2} \\
& \leqslant\left(t_{2}-t_{1}\right)^{1+\beta} m, \quad m:=2^{1 / 2}(3 c)^{\beta} \kappa^{\beta} .
\end{aligned}
$$

2. Fix an arbitrary $t \in(0, T]$. For every $h \in(0, t]$ and $\hat{h} \in[h / 2, h]$,

$$
\begin{aligned}
& |\mathbf{e}(t-h, t)-\mathbf{e}(t-\hat{h}, t)| \\
\leqslant & \frac{1}{\hat{h}}\left(\int_{t-\hat{h}}^{t}\left|\mathbf{e}(t-h, t)-\frac{\dot{\mathbf{x}}(s)}{|\dot{\mathbf{x}}(s)|}\right| d s+\int_{t-\hat{h}}^{t}\left|\mathbf{e}(t-\hat{h}, t)-\frac{\dot{\mathbf{x}}(s)}{|\dot{\mathbf{x}}(s)|}\right| d s\right) \\
\leqslant & \frac{1}{\hat{h}}\left\{m h^{1+\beta}+m \hat{h}^{1+\beta}\right\} \leqslant 3 m h^{\beta} .
\end{aligned}
$$

Define

$$
\mathbf{n}(t-):=\mathbf{e}(0, t)+\sum_{i=0}^{\infty}\left\{\mathbf{e}\left(t-2^{-i-1} t, t\right)-\mathbf{e}\left(t-2^{-i} t, t\right)\right\} .
$$

For every fixed $h \in(0, t]$, let $j$ be an integer such that $h \in\left[t / 2^{j+1}, t / 2^{j}\right]$. Then

$$
\begin{array}{r}
\mathbf{n}(t-)-\mathbf{e}(t-h, t) \\
=\mathbf{e}\left(t-2^{-j-1} t, t\right)+\sum_{i=j+1}^{\infty}\left\{\mathbf{e}\left(t-2^{-i-1} t, t\right)-\mathbf{e}\left(t-2^{-i} t, t\right)\right\}-\mathbf{e}(t-h, t) .
\end{array}
$$


It then follows that

$$
\begin{aligned}
|\mathbf{n}(t-)-\mathbf{e}(t-h, t)| & \leqslant 3 m \sum_{i=j+1}^{\infty}\left(\frac{t}{2^{i}}\right)^{\beta}+3 m h^{\beta} \\
& =\frac{3 m}{1-2^{-\beta}}\left(\frac{t}{2^{j+1}}\right)^{\beta}+3 m h^{\beta} \\
& \leqslant \frac{6 m h^{\beta}}{1-2^{-\beta}}
\end{aligned}
$$

Therefore,

$$
\mathbf{n}(t-):=\lim _{h \searrow 0} \mathbf{e}(t-h, t)
$$

for every $t \in(0, T]$.

Similarly, for every $t \in[0, T)$, define

$$
\mathbf{n}(t+)=\mathbf{e}(t, T)+\sum_{i=0}^{\infty}\left\{\mathbf{e}\left(t, t+(T-t) 2^{-i-1}\right)-\mathbf{e}\left(t, t+(T-t) 2^{-i}\right)\right\} .
$$

Then

$$
\mathbf{n}(t+)=\lim _{h \searrow 0} \mathbf{e}(t, t+h) .
$$

Therefore, for every $0 \leqslant t_{1}<t_{2} \leqslant T$, setting $h=t_{2}-t_{1}$ we have

$$
\begin{aligned}
& \mathbf{n}\left(t_{1}+\right)=\mathbf{e}\left(t_{1}, t_{2}\right)+\sum_{i=1}^{\infty}\left\{\mathbf{e}\left(t_{1}, t_{1}+2^{-i-1} h\right)-\mathbf{e}\left(t_{1}, t_{1}+2^{-i} h\right)\right\} \\
& \mathbf{n}\left(t_{2}-\right)=\mathbf{e}\left(t_{1}, t_{2}\right)+\sum_{i=1}^{\infty}\left\{\mathbf{e}\left(t_{2}-2^{-i-1} h, t_{2}\right)-\mathbf{e}\left(t_{2}-2^{-i} h, t_{2}\right)\right\}
\end{aligned}
$$

It then follows that

$$
\begin{gathered}
\left|\mathbf{n}\left(t_{1}+\right)-\mathbf{e}\left(t_{1}, t_{2}\right)\right| \leqslant \frac{3 m\left(t_{2}-t_{1}\right)^{\beta}}{1-2^{-\beta}}, \\
\left|\mathbf{n}\left(t_{2}-\right)-\mathbf{e}\left(t_{1}, t_{2}\right)\right| \leqslant \frac{3 m\left(t_{2}-t_{1}\right)^{\beta}}{1-2^{-\beta}} .
\end{gathered}
$$

Finally, when $t \in(0, T)$ and $h \in(0, \min \{t, T-t\}]$,

$$
\begin{aligned}
|\mathbf{n}(t+)-\mathbf{n}(t-)| \leqslant|\mathbf{n}(t+)-\mathbf{e}(t, t+h)| & +|\mathbf{e}(t, t+h)-\mathbf{e}(t-h, t+h)| \\
& +|\mathbf{e}(t-h, t+h)-\mathbf{e}(t-h, t)|+|\mathbf{e}(t-h, t)-\mathbf{n}(t-)| .
\end{aligned}
$$

Sending $h \rightarrow 0$ we conclude that $\mathbf{n}(t-)=\mathbf{n}(t+)$. Now, we can define $\mathbf{n}:[0, T] \rightarrow \mathbb{S}^{n-1}$ by $\mathbf{n}(0)=\mathbf{n}(0+), \mathbf{n}(t)=\mathbf{n}(t \pm)$ for $t \in(0, T)$ and $\mathbf{n}(T)=\mathbf{n}(T-)$. Then for $0 \leqslant t_{1}<t_{2} \leqslant T$,

$$
\begin{aligned}
\left|\mathbf{n}\left(t_{2}\right)-\mathbf{n}\left(t_{1}\right)\right| & \leqslant\left|\mathbf{n}\left(t_{2}\right)-\mathbf{e}\left(t_{1}, t_{2}\right)\right|+\left|\mathbf{n}\left(t_{1}\right)-\mathbf{e}\left(t_{1}, t_{2}\right)\right| \\
& \leqslant \frac{6 m}{1-2^{-\beta}}\left(t_{2}-t_{1}\right)^{\beta}
\end{aligned}
$$


Note that

$$
\frac{6 m}{1-2^{-\beta}}=\frac{6 * 2^{\frac{1}{2}}(3 c)^{\frac{\beta}{2}} \kappa^{\beta}}{1-2^{-\beta}} \leqslant \frac{15 c^{\frac{\beta}{2}}}{1-2^{-\beta}} \kappa^{\beta}=M \kappa^{\beta},
$$

where

$$
M:=\frac{15 c^{\frac{\beta}{2}}}{1-2^{-\beta}} .
$$

Finally, since $\mathbf{x}$ is Lipschitz continuous, we see that $\mathbf{n}(s)=\dot{\mathbf{x}}(s) /|\dot{\mathbf{x}}(s)|$ and $\dot{\mathbf{x}}(s)=\mathbf{n}(s) v(\mathbf{x}(s), s)$ for a.e. $s \in(0, T)$. Upon using the integral equation

$$
\mathbf{x}(t)-\mathbf{x}(0)=\int_{0}^{t} \dot{\mathbf{x}}(s) d s=\int_{0}^{t} \mathbf{n}(s) v(\mathbf{x}(s), s) d s, \quad \forall t \in[0, T]
$$

we then conclude that $\dot{\mathbf{x}}(t)=\mathbf{n}(t) v(\mathbf{x}(t), t)$ for all $t \in[0, T]$. This completes the proof of Theorem 2.

REMARK 3.1 (1) From Example 6, we see that the exponent $\beta$ in the Theorem is optimal.

(2) The length of the trajectory $\ell\left(x\left[t_{1}, t_{2}\right]\right)$ and the line distance $l=\left|x\left(t_{2}\right)-x\left(t_{1}\right)\right|$ between end points are shown in [34] to have the relation $\ell=l+O\left(l^{1+\alpha}\right)$. Here we can compute

$$
\begin{aligned}
\ell\left(\mathbf{x}\left(\left[t_{1}, t_{2}\right]\right)-l\right. & =\int_{t_{1}}^{t_{2}}|\dot{\mathbf{x}}(s)| d s-\left|\mathbf{x}\left(t_{2}\right)-\mathbf{x}\left(t_{1}\right)\right| \leqslant \int_{t_{1}}^{t_{2}}(|\dot{\mathbf{x}}|-|\dot{\mathbf{y}}|) d s \\
& =\int_{t_{1}}^{t_{2}}(v(\mathbf{x}, s)-v(\mathbf{y}, s)) d s \leqslant \kappa^{\alpha} \int_{t_{1}}^{t_{2}}|\mathbf{x}-\mathbf{y}|^{\alpha} d s \\
& \leqslant \kappa^{\alpha}\left(t_{2}-t_{1}\right)\left[3 c\left(t_{2}-t_{1}\right)^{2}\right]^{\alpha /(2-\alpha)} \kappa^{\alpha \beta} \leqslant(3 c)^{\beta} \kappa^{2 \beta} l^{1+2 \beta},
\end{aligned}
$$

since

$$
l=\left|x\left(t_{2}\right)-x\left(t_{1}\right)\right| \geqslant \int_{t_{1}}^{t_{2}}|\dot{\mathbf{y}}| d s \geqslant\left|t_{2}-t_{1}\right| .
$$

Thus, $\ell=l+O\left(l^{1+2 \beta}\right)$, optimal by Example 6 .

\section{A clearing lemma}

To show that $\mathrm{D}(t)$ has Lipschitz boundary $\Gamma(t)$, it is sufficient to show that at every $p \in \Gamma(t), \mathrm{D}(t)$ contains a cone with fixed open angle and vertex $p$. In the sequel, we shall prove a stronger result: in a small ball centered at $p, \mathrm{D}(t)$ contains a region that is on or below a $C^{1+\alpha / 2}$ graph in the direction $\dot{\mathbf{x}}(t) /|\dot{\mathbf{x}}(t)|$ and containing $p$, where $\mathbf{x} \in \mathrm{C}_{b}(t)$ is any element satisfying $\mathbf{x}(t)=p$. This implies that at every boundary point, $\mathrm{D}(t)$ contains a cone of open angle that can be arbitrarily close to $\pi$. For convenience, we set

$$
M_{1}:=\frac{3}{2}+M=\frac{3}{2}+\frac{15 c^{\beta / 2}}{1-2^{-\beta}} .
$$

Theorem 3 Assume (2.1) and (3.1). Let $T>0$ and $\mathbf{x} \in \mathrm{C}_{b}(T)$. Set $p=\mathbf{x}(t)$ and $\mathbf{n}=\dot{\mathbf{x}}(t) /|\dot{\mathbf{x}}(t)|$. Then

$$
\begin{gathered}
\left\{z \in B\left(p, \kappa^{\beta} t^{1+\beta}\right):(z-p) \cdot \mathbf{n} \leqslant-M_{1} \kappa^{\frac{\alpha}{2}}|z-p|^{1+\frac{\alpha}{2}}\right\} \subset \mathrm{D}(t), t \in(0, T] \\
\left\{z \in B\left(p, \kappa^{\beta}(T-t)^{1+\beta}\right):(z-p) \cdot \mathbf{n}>M_{1} \kappa^{\frac{\alpha}{2}}|z-p|^{1+\frac{\alpha}{2}}\right\} \subset \mathbb{R}^{n} \backslash \mathrm{D}(t), t \in[0, T) .
\end{gathered}
$$


Consequently, for every $t \in(0, T), \dot{\mathbf{x}}(t) /|\dot{\mathbf{x}}(t)|$ is the unit outward normal to $\mathrm{D}(t)$ at $\mathbf{x}(t)$.

REMARK 4.1 Here in the second inclusion, although $t \in[0, T)$, this is not a statement about $D_{0}$ because points in $\partial D_{0}$ need not be on a boundary characteristic.

Proof. Clearly, the last assertion is a direct consequence of the two inclusions.

To prove the first assertion, we need only consider the case $t=T$. The basic idea of the proof is as follows, cf. Figure 2(a). For any unit vector $\mathbf{e}$ that is perpendicular to $\mathbf{n}(T)$ and for every $s \in[0, T)$ such that $T-s$ is small, we construct a curve $\mathbf{y}(\mathbf{e}, s ; \cdot)$ in $\mathrm{C}(T)$ which coincides with $\mathbf{x}(\cdot)$ on $[0, s]$, but gradually diverges from $\mathbf{x}$ in $(s, T]$ in the direction $\mathbf{e}$. This construction tells us that $\mathbf{y}(\mathbf{e}, s ; t) \in \mathrm{D}(T)$ for all $t \in[s, T]$. Taking all possible $s$ and $\mathbf{e}$, we then know that $\mathrm{D}(T)$ will contain a fan like region as depicted in Figure 2(a). Careful arrangement shows that the "end" of the fan $\mathbf{y}(\cdot, \cdot, T)$ is a $C^{1+\alpha / 2}$ graph, in the direction $\mathbf{n}(T)$. The calculation is simple when $\mathbf{e}$ is taken as a general unit vector. In Figure 2(b), we show the exact solution explained in Example 6. The calculation there shows that the exponent $1+\alpha / 2$ in our Theorem is optimal.

(i) Set $p=\mathbf{x}(T)$ and $\mathbf{n}(t):=\dot{\mathbf{x}}(t) /|\dot{\mathbf{x}}(t)|$. Let $\mathbf{e} \in \mathbb{S}^{n-1}$ be any unit vector and $s \in[0, T]$. Consider

$$
\mathbf{y}(t)= \begin{cases}\mathbf{x}(t) & \text { if } t \in[0, s], \\ \mathbf{x}(t)+\kappa^{\beta}(T-s)^{\beta}(t-s) \mathbf{e} & \text { if } t \in(s, T] .\end{cases}
$$

Since $\dot{\mathbf{x}}(t)=v(\mathbf{x}(t), t) \mathbf{n}(t)$, when $t \in[s, T]$, we have

$$
\begin{aligned}
|\dot{\mathbf{y}}(t)|^{2} & =\left|v(\mathbf{x}(t), t) \mathbf{n}(t)+\kappa^{\beta}(T-s)^{\beta} \mathbf{e}\right|^{2} \\
& =v^{2}(\mathbf{x}, t)+\kappa^{2 \beta}(T-s)^{2 \beta}+2 v(\mathbf{x}, t) \kappa^{\beta}(T-s)^{\beta} \mathbf{n}(t) \cdot \mathbf{e} .
\end{aligned}
$$

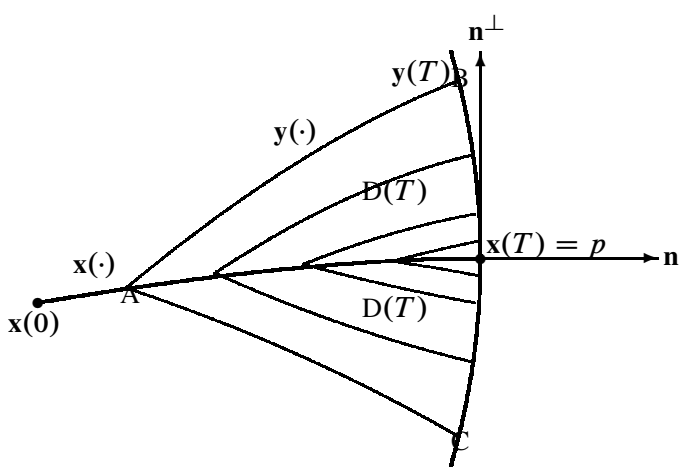

(a)

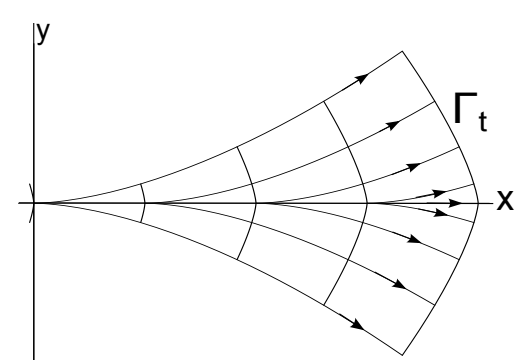

(b)

FIG. 2. (a) A schematic depiction of a few examples of the trajectory $\mathbf{y}(\mathbf{e}, s ; \cdot)$ with different choices of $s$. The union of all these curves gives a subset of $\mathrm{D}(T)$, looking like a fan with vertices $A, B, C$.

(b) Exact solutions of the characteristic trajectories and fronts initiated from the origin from Example 6. All fronts in the region have the form $x=t-|y|^{1+\alpha / 2}$. The boundary characteristic trajectories have the form $|y|=\left[\left(1-\alpha^{2} / 4\right) \max \{x-\right.$ $\left.\left.t_{0}, 0\right\}\right]^{1+\beta}$. 
Note that when $t \in[s, T]$,

$$
\begin{aligned}
\mathbf{n}(t) \cdot \mathbf{e}=(\mathbf{n}(t)-\mathbf{n}(T)) \cdot \mathbf{e}+\mathbf{n}(T) \cdot \mathbf{e} \leqslant M \kappa^{\beta}|T-s|^{\beta}+\mathbf{n}(T) \cdot \mathbf{e} \\
\begin{aligned}
v^{2}(\mathbf{x}(t), t)-v^{2}(\mathbf{y}(t), t) & \leqslant 2 v(\mathbf{x}, t)(v(\mathbf{x}, t)-v(\mathbf{y}, t)) \\
& \leqslant 2 v(\mathbf{x}, t) \kappa^{\alpha}|\mathbf{x}-\mathbf{y}|^{\alpha} \leqslant 2 v(\mathbf{x}, t) \kappa^{2 \beta}(T-s)^{2 \beta}
\end{aligned}
\end{aligned}
$$

since $|\mathbf{x}(t)-\mathbf{y}(t)| \leqslant \kappa^{\beta}(T-s)^{1+\beta}$ and $(1+\beta) \alpha=2 \beta$. Thus, for all $t \in[s, T]$,

$$
|\dot{\mathbf{y}}|^{2}-v^{2}(\mathbf{y}, t) \leqslant 2 v(\mathbf{x}, t) \kappa^{2 \beta}(T-s)^{2 \beta}\left\{1+\frac{1}{2 v(\mathbf{x}, t)}+M+\frac{\mathbf{n}(T) \cdot \mathbf{e}}{\kappa^{\beta}(T-s)^{\beta}}\right\} .
$$

Now assume that $\mathbf{e}$ satisfies

$$
\frac{\mathbf{n}(T) \cdot \mathbf{e}}{\kappa^{\beta}(T-s)^{\beta}} \leqslant-M-\frac{3}{2} \equiv-M_{1} .
$$

Then, $|\dot{\mathbf{y}}| \leqslant v(\mathbf{y}, t)$ for all $t \in[t, T]$. This implies that $\mathbf{y} \in \mathrm{C}(T)$ and $\mathbf{y}(0) \in \mathrm{D}_{0}$. Hence,

$$
\mathbf{y}(T)=\mathbf{x}(T)+\kappa^{\beta}(T-s)^{1+\beta} \mathbf{e}=p+\kappa^{\beta}(T-s)^{1+\beta} \mathbf{e} \in \mathrm{D}(T) .
$$

Now let $z \in B\left(p, \kappa^{\beta} T^{1+\beta}\right)$ be an arbitrary point. Define $s \in[0, T]$ by $\kappa^{\beta}(T-s)^{1+\beta}=|z-p|$, e by $\mathbf{e}=(z-p) /|z-p|$, and $\mathbf{y}$ as above. Then $\mathbf{y}(T)=z$. Also,

$$
\frac{\mathbf{n}(T) \cdot \mathbf{e}}{\kappa^{\beta}(T-s)^{\beta}}=\frac{\mathbf{n}(T)}{\kappa^{\beta}(T-s)^{\beta}} \cdot \frac{z-p}{|z-p|}=\frac{\mathbf{n} \cdot(z-p)}{\kappa^{\alpha / 2}|z-p|^{1+\alpha / 2}} .
$$

Thus, according to the previous calculation, $z=\mathbf{y}(T) \in \mathrm{D}(t)$ if

$$
(z-p) \cdot \mathbf{n}(T) \leqslant-M_{1} \kappa^{\alpha / 2}|z-p|^{1+\alpha / 2} .
$$

This first assertion of the Theorem thus follows.

(ii) Let's fix $s \in[0, T)$. Given $\mathbf{e} \in \mathbb{S}^{n-1}$ and $\hat{T} \in(s, T]$ consider the function

$$
\mathbf{y}(t)=\mathbf{x}(t)+\kappa^{\beta}(\hat{T}-s)^{\beta}(\hat{T}-t) \mathbf{e}, \quad \forall t \in[s, \hat{T}] .
$$

Using the same calculation as above except using the revised estimate

$$
\begin{aligned}
-\mathbf{n}(t) \cdot \mathbf{e} & =-\mathbf{n}(s) \cdot \mathbf{e}+(\mathbf{n}(s)-\mathbf{n}(t)) \cdot \mathbf{e} \\
& \leqslant-\mathbf{n}(s) \cdot \mathbf{e}+M \kappa^{\beta}(\hat{T}-s)^{\beta}
\end{aligned}
$$

we can show that $|\dot{\mathbf{y}}(t)|<v(\mathbf{y}(t), t)$ for all $t \in[s, \hat{T}]$ if

$$
\frac{\mathbf{n}(s) \cdot \mathbf{e}}{\kappa^{\beta}(\hat{T}-s)^{\beta}}>M_{1}
$$

Assume this is true. Then there exists $\varepsilon>0$ such that

$$
|\dot{\mathbf{y}}(t)| \leqslant(1-\varepsilon) v(\mathbf{y}(t), t) \text { for all } t \in[s, \hat{T}] .
$$


This implies that $\mathbf{y}(s) \notin \Gamma(s)$ since otherwise it would imply that $\mathbf{y}(\hat{T})$ is an interior point of $\mathrm{D}(\hat{T})$, contradicting the fact that $\mathbf{y}(\hat{T})=\mathbf{x}(\hat{T}) \in \Gamma(\hat{T})$.

Now for any given $z \in B\left(\mathbf{x}(s), \kappa^{\beta}(T-s)^{1+\beta}\right)$ satisfying

$$
(z-\mathbf{x}(s)) \cdot \mathbf{n}(s)>M_{1} \kappa^{\alpha / 2}|z-\mathbf{x}(s)|^{1+\alpha / 2},
$$

set

$$
\mathbf{e}=(z-\mathbf{x}(s)) /|z-\mathbf{x}(s)| \text { and } \hat{T}=s+\left[|z-\mathbf{x}(s)| / \kappa^{\alpha}\right]^{1 /(1+\beta)} .
$$

Then the above defined $\mathbf{y}$ gives $\mathbf{y}(s)=z \notin \mathrm{D}(s)$. This completes the proof.

\section{Lipschitz continuity of $\Gamma(t)$}

In this section, we study the regularity of the front $\Gamma(t)$. We shall show that in any small enough ball, $\Gamma(t)$ is the union of finitely many Lipschitz graphs with a uniform Lipschitz constant. If we allow the smallness of the ball to depend on $t$, then the number of Lipschitz graphs is at most $2 n$ where $n$ is the space dimension; we call this a local estimate. To obtain a global estimate, i.e., to obtain a uniform positive size on the radii of these balls, we assume that $\partial \mathrm{D}_{0}$ satisfies an interior cone condition.

\subsection{Local estimate}

To describe graphs, it is convenient to use cubes. Hence we introduce

$$
\begin{aligned}
C(q, \rho) & :=\left\{q+\left(x_{1}, \cdots, x_{n}\right) \mid x_{i} \in[-\rho, \rho] \quad \forall i=1, \cdots, n\right\}, \quad \forall q \in \mathbb{R}^{n}, \rho>0, \\
\check{x}_{i} & :=\left(x_{1}, \cdots, x_{i-1}, x_{i+1}, \cdots, x_{n}\right), \quad \forall\left(x_{1}, \cdots, x_{n}\right) \in \mathbb{R}^{n}, i=1, \cdots, n .
\end{aligned}
$$

TheOREM 4 Assume (2.1) and (3.1). Let $t>0$ and define

$$
r=r(t):=\min \left\{\frac{\kappa^{\beta} t^{1+\beta}}{2 \sqrt{n}}, \frac{R_{0}}{\kappa}\right\}, \quad R_{0}:=n^{-\frac{1}{2}-\frac{1}{\alpha}}\left[9+\frac{90 c^{\beta / 2}}{1-2^{-\beta}}\right]^{-2 / \alpha}
$$

Then for any $q \in \Gamma(t)$ there are functions $\psi_{1}^{ \pm}, \cdots, \psi_{n}^{ \pm}$defined on $\mathbb{R}^{n-1}$ such that the following holds:

$$
\begin{gathered}
\left|\psi_{i}^{s}\left(x^{\prime}\right)-\psi_{i}^{s}\left(y^{\prime}\right)\right| \leqslant 2 \sqrt{n}\left|x^{\prime}-y^{\prime}\right|, \quad \forall x^{\prime}, y^{\prime} \in \mathbb{R}^{n-1}, i=1, \cdots, n, s=+,-, \\
\Gamma(t) \cap C(q, r) \subset \bigcup_{i=1}^{n}\left\{\left(x_{1}, \cdots, x_{n}\right) \in \mathbb{R}^{n} \mid x_{i}=\psi_{i}^{+}\left(\check{x}_{i}\right) \text { or } x_{i}=\psi_{i}^{-}\left(\check{x}_{i}\right)\right\}, \\
\mathrm{D}(t) \supset \bigcup_{i=1}^{n}\left\{\left(x_{1}, \cdots, x_{n}\right) \in C(q, r) \mid x_{i} \geqslant \psi_{i}^{+}\left(\check{x}_{i}\right) \text { or } x_{i} \leqslant \psi^{-}\left(\check{x}_{i}\right)\right\} .
\end{gathered}
$$

Proof. We divide the proof into several steps.

1. For each $p \in \Gamma(t)$, we define $\mathbf{N}(p)$, called the set of normals of $\Gamma(t)$ at $p$ by

$$
\mathbf{N}(p):=\left\{\frac{\dot{\mathbf{x}}(t)}{|\dot{\mathbf{x}}(t)|} \mid \mathbf{x} \in \mathrm{C}_{b}(t), \mathbf{x}(t)=p\right\}
$$


This set is none-empty and well-defined since every point on $\partial \mathrm{Q}$ lies on at least one boundary characteristic curve and each boundary characteristic curve has positive speeds and continuous directions. Also, there is no need to indicate that $\mathbf{N}(p)$ depends on $t$ since $t=\tau(p)$.

Next, denote by $\mathbf{e}_{1}, \cdots, \mathbf{e}_{n}$ the unit coordinate vectors of $\mathbb{R}^{n}$. We define

$$
\Gamma_{i}^{ \pm}(t)=\left\{p \in \Gamma(t) \mid \exists \mathbf{n} \in \mathbf{N}(p), \quad \mp \mathbf{n} \cdot \mathbf{e}_{i} \geqslant \frac{1}{\sqrt{n}}\right\}, \quad i=1, \cdots, n .
$$

Clearly,

$$
\Gamma(t)=\bigcup_{i=1}^{n}\left(\Gamma_{i}^{+}(t) \cup \Gamma_{i}^{-}(t)\right) .
$$

2. Let $r$ be a positive constant to be specified later. Let $q=\left(q_{1}, \cdots, q_{n}\right) \in \Gamma(t)$ be an arbitrary point. We shall show that each $\Gamma_{i}^{ \pm}(t) \cap C(q, r)$ lies on a Lipschitz graph of the form $x_{i}=\psi_{i}^{ \pm}\left(\check{x}_{i}\right)$.

Let's consider $\Gamma_{1}^{-}(t) \cap C(q, r)$. If it is an empty set, we define $\psi_{i}^{-}\left(x^{\prime}\right) \equiv q_{i}-r$ for all $x^{\prime} \in \mathbb{R}^{n-1}$. If it is non-empty, we proceed as follows.

Let $p=\left(p_{1}, p^{\prime}\right) \in \Gamma_{1}^{-}(t) \cap C(q, r)$ be arbitrary. Set

$$
\psi\left(p ; x^{\prime}\right)=p_{1}-2 \sqrt{n}\left|p^{\prime}-x^{\prime}\right|, \quad \forall x^{\prime} \in \mathbb{R}^{n-1} .
$$

Regarding the $x_{1}$-axis as the vertical axis, we shall use Theorem 3 to show that in $C(q, r)$, the set under the graph $x_{1}=\psi\left(p ; x^{\prime}\right)$ is in $\mathrm{D}(t)$. For this purpose, let $x=\left(x_{1}, x^{\prime}\right) \in C(q, r)$ be an arbitrary point satisfying

$$
x_{1} \leqslant \psi\left(p ; x^{\prime}\right) \text {. }
$$

Since $p \in \Gamma_{1}^{-}(t)$, there exists $\mathbf{n} \in \mathbf{N}(p)$ such that $\mathbf{n} \cdot \mathbf{e}_{1} \geqslant 1 / \sqrt{n}$. Hence, to show $\left(x_{1}, x^{\prime}\right) \in \mathrm{D}(t)$, by the "clear out" Lemma (Theorem 3 ) we need only verify two conditions:

$$
\text { (i) }|x-p| \leqslant \kappa^{\beta} t^{1+\beta}, \quad \text { (ii) } \quad(p-x) \cdot \mathbf{n} \geqslant M_{1} \kappa^{\alpha / 2}|x-p|^{1+\alpha / 2} \text {. }
$$

For condition (i), we need only $2 \sqrt{n} r \leqslant \kappa^{\beta} t^{1+\beta}$ since both $x$ and $p$ is in $C\left(x^{0}, r\right)$.

To check condition (ii), we use $\mathbf{n} \cdot \mathbf{e}_{1} \geqslant 1 / \sqrt{n}$ and $p_{1}=\psi\left(p ; x^{\prime}\right)+2 \sqrt{n}\left|x^{\prime}-p^{\prime}\right|$ :

$$
\begin{aligned}
(p-x) \cdot \mathbf{n} & =\left(p_{1}-x_{1}\right) \mathbf{e}_{1} \cdot \mathbf{n}+\left(0, p^{\prime}-x^{\prime}\right) \cdot \mathbf{n} \\
& =\left\{\psi\left(p ; x^{\prime}\right)-x_{1}+2 \sqrt{n}\left|p^{\prime}-x^{\prime}\right|\right\} \mathbf{e}_{1} \cdot \mathbf{n}+\left(0, p^{\prime}-x^{\prime}\right) \cdot \mathbf{n} \\
& \geqslant \frac{\psi\left(p ; x^{\prime}\right)-x_{1}}{\sqrt{n}}+\left|p^{\prime}-x^{\prime}\right| ; \\
|p-x|^{1+\alpha / 2} & \leqslant(2 \sqrt{n} r)^{\alpha / 2}\left(\left|p_{1}-x_{1}\right|+\left|p^{\prime}-x^{\prime}\right|\right) \\
& =(2 \sqrt{n} r)^{\alpha / 2}\left(\psi\left(p ; x^{\prime}\right)-x_{1}+(1+2 \sqrt{n})\left|x^{\prime}-p^{\prime}\right|\right) .
\end{aligned}
$$

Hence, to have $(p-x) \cdot \mathbf{n} \geqslant M_{1} \kappa^{\alpha / 2}|x-p|^{1+\alpha / 2}$, if suffices to have

$$
(2 \sqrt{n} r)^{\alpha / 2} M_{1} \kappa^{\alpha / 2} \leqslant \frac{1}{3 \sqrt{n}} .
$$

In conclusion both conditions (i) and (ii) are satisfied if we take

$$
r=r(t):=\min \left(\frac{\kappa^{\beta} t^{1+\beta}}{2 \sqrt{n}}, \frac{R_{0}}{\kappa}\right), \quad R_{0}:=\frac{1}{\sqrt{n}\left(6 \sqrt{n} M_{1}\right)^{2 / \alpha}} .
$$


Substituting the expression $M_{1}$ given in the previous section, we obtain the expression $R_{0}$ in the statement of the Theorem.

3. One can verify that $\Gamma_{1}^{-}(t)$ is a compact set. Hence, we define

$$
\psi_{1}^{-}\left(x^{\prime}\right):=\max \left\{\psi\left(p ; x^{\prime}\right) \mid p \in \Gamma_{1}^{-}(t) \cap C(q, r)\right\}, \quad \forall x^{\prime} \in \mathbb{R}^{n-1} .
$$

Such a function has the following properties:

(a) $\psi_{1}^{-}$is Lipschitz continuous with Lipschitz constant $2 \sqrt{n}$. This follows from the fact that each $\psi(p ; \cdot)$ has the said property.

(b) In the cube $C(q, r)$, the region below the graph $x_{1}=\psi_{1}^{-}\left(x^{\prime}\right)$ belongs to $\mathrm{D}(t)$ :

$$
\left(x_{1}, x^{\prime}\right) \in C(q, r), \quad x_{1} \leqslant \psi_{1}^{-}\left(x^{\prime}\right) \quad \Longrightarrow \quad\left(x_{1}, x^{\prime}\right) \in \mathrm{D}(t) .
$$

(c) If $\left(x_{1}, x^{\prime}\right) \in \Gamma_{1}^{-}(t) \cap C(q, r)$, then $x_{1}=\psi_{1}^{-}\left(x^{\prime}\right)$. This follows from the definition of $\psi_{1}^{-}$and its property.

Repeating the analogous process for the remaining sets $\Gamma_{i}^{ \pm}(t) \cap C(q, r)$, we then obtain Lipschitz continuous functions $\psi_{i}^{ \pm}$with Lipschitz constant $2 \sqrt{n}$ and the following properties:

$$
\begin{aligned}
\Gamma_{i}^{ \pm}(t) \cap C(q, r) & \subset\left\{x \in \mathbb{R}^{n} \mid x_{i}=\psi_{i}^{ \pm}\left(\check{x}_{i}\right)\right\}, \\
D(t) & \supset\left\{x \in C(q, r) \mid x_{i} \leqslant \psi_{i}^{-}\left(\check{x}_{i}\right) \text { or } x_{i} \geqslant \psi_{i}^{+}\left(\check{x}_{i}\right)\right\} .
\end{aligned}
$$

Finally, since $\Gamma(t) \subset \cup_{i=1}^{n}\left(\Gamma_{i}^{+}(t) \cup \Gamma_{i}^{-}(t)\right)$ the assertion of the theorem thus follows.

\subsection{Global estimates}

The size $r(t)$ in the local estimate in Theorem 4 is small when $t$ is small. Here we investigate the case when $t$ is small. For this we assume that $\mathrm{D}_{0}$ satisfies the interior cone condition stated as below:

There exist constants $\theta_{0} \in(0, \pi / 2)$ and $\delta_{0}>0$ and a function $\mathbf{n}_{0}: \Gamma_{0} \rightarrow \mathbb{S}^{n-1}$ such that

$$
\left\{x \in C\left(p, \delta_{0}\right)\left|(x-q) \cdot \mathbf{n}_{0}(p) \leqslant-\right| x-q \mid \cos \theta_{0}\right\} \subset D_{0} \quad \forall p \in \Gamma_{0} .
$$

EXAMPLE 7 The interior cone condition is satisfies by a set being the union of eight sectors:

$$
\mathrm{D}_{0}:=\left\{(\rho \cos \theta, \rho \cos \theta) \in \mathbb{R}^{2}|0 \leqslant \rho \leqslant 1,5| \sin (4 \theta) \mid \leqslant 1\right\} .
$$

Theorem 5 Assume (2.1), (3.1), and (5.1). Define

$$
R_{1}:=\min \left\{\frac{\delta_{0}}{2}, \frac{M_{4}}{\kappa}\right\}, \quad M_{4}:=\frac{1}{2}\left(\frac{c}{9} \sin ^{2} \frac{\theta_{0}}{2}\right)^{\frac{1}{\alpha}}\left(\frac{1}{c}\right)^{\frac{1}{2}}\left(\frac{2^{\beta}-1}{15}\right)^{\frac{1}{\beta}} .
$$

Then for every $t>0$ and $q \in \Gamma(t), \Gamma(t) \cap B\left(p ; R_{1}\right)$ is contained in a union of at most $N=\left(32 \pi / \theta_{0}\right)^{n-1}$ Lipschitz graphs where each graph can be represented, after a rotation, as $x_{1}=\psi\left(x_{2}, \cdots, x_{n}\right)$ where $\psi$ is a Lipschitz function with Lipschitz constant no bigger than $\cot \frac{\theta_{0}}{4}$.

The key to the proof relies on the following supplement to Theorem 3.

LEMma 5.1 For every $T>0$ and every $q \in \Gamma(T)$, there exists $\mathbf{n}_{0}(q) \in \mathbb{S}^{n-1}$ such that

$$
\left\{z \in B\left(q, 2 R_{1}\right)\left|(z-q) \cdot \mathbf{n}_{0}(q) \leqslant-\right| x-q \mid \cos \frac{\theta_{0}}{2}\right\} \subset \mathrm{D}(T) .
$$


Proof. For convenience, we define

$$
\varepsilon:=\frac{2}{3} \sin \frac{\theta_{0}}{2}, L:=\min \left\{\delta_{0}, \frac{1}{\kappa}\left(\frac{\varepsilon}{3} \sin \frac{\theta_{0}}{2}\right)^{1 / \alpha}, \frac{\varepsilon}{\kappa}\left(\frac{1}{3 M} \sin \frac{\theta_{0}}{2}\right)^{1 / \beta}\right\}, t_{1}:=\frac{L}{\varepsilon} .
$$

One can verify that this $L=2 R_{1}$.

Let $\mathbf{x} \in \mathbb{C}_{b}(T)$ satisfy $\mathbf{x}(T)=q$. Set $p=\mathbf{x}(0)$ and $\mathbf{n}(t)=\dot{\mathbf{x}}(t) /|\dot{\mathbf{x}}(t)|$ for every $t \in[0, T]$. Also, let $\mathbf{n}_{0}(p)$ be the unit vector satisfying (5.1). By the second inclusion in Theorem 3 with $t=0$ and a simple trigonometry we can show that

$$
\mathbf{n}(0) \cdot \mathbf{n}_{0}(p) \geqslant \sin \theta_{0} .
$$

Since $T=\tau(q)$, we define

$$
\mathbf{n}_{0}(q)= \begin{cases}\mathbf{n}_{0}(p) & \text { if } \tau(q) \in\left(0, t_{1}\right) \\ \mathbf{n}(\tau(q)) & \text { if } \tau(q) \geqslant t_{1}\end{cases}
$$

Let $z \in B(q, L)$ be an arbitrary point that satisfies

$$
(z-q) \cdot \mathbf{n}_{0}(q) \leqslant-|z-q| \cos \frac{\theta_{0}}{2} .
$$

We want to show that $z \in \mathrm{D}(T)$. For this set $l=|z-q|$ and $\mathbf{e}=(z-q) /|z-q|$ so that

$$
z=q+l \mathbf{e}, \quad 0 \leqslant l \leqslant L, \quad|\mathbf{e}|=1, \quad \mathbf{e} \cdot \mathbf{n}_{0}(q) \leqslant-\cos \frac{\theta_{0}}{2} .
$$

Consider the function

$$
\mathbf{y}(t)=\mathbf{x}(t)+(l-\varepsilon[T-t]) \mathbf{e}, \quad \forall t \in[\tilde{t}, T], \quad \tilde{t}:=\max \{0, T-l / \varepsilon] .
$$

Note that $\mathbf{y}(T)=\mathbf{x}(T)+l \mathbf{e}=z$. Also $\mathbf{y}(\tilde{t}) \in \mathrm{D}(\tilde{t})$, by the following reasons. When $T \geqslant l / \varepsilon$ we have $\mathbf{y}(\tilde{t})=\mathbf{x}(\tilde{t}) \in \mathrm{D}(\tilde{t})$. When $T<l / \varepsilon$, we have $\tilde{t}=0, \mathbf{x}(0)=p, T<L / \varepsilon=t_{1}$, $\mathbf{n}_{0}(q)=\mathbf{n}_{0}(p)$, and

$$
\frac{(\mathbf{y}(0)-p)}{|\mathbf{y}(0)-p|} \cdot \mathbf{n}_{0}(p)=\mathbf{e} \cdot \mathbf{n}_{0}(q) \leqslant-\cos \frac{\theta_{0}}{2}<-\cos \theta_{0} .
$$

Since

$$
|\mathbf{y}(0)-p|=l-\varepsilon T \leqslant l \leqslant \delta_{0},
$$

it follows from (5.1) that $\mathbf{y}(0) \in \mathrm{D}_{0}$. Hence, $\mathbf{y}(\tilde{t}) \in \mathrm{D}(\tilde{t})$.To complete the proof, it is sufficient to show that $|\dot{\mathbf{y}}(t)| \leqslant v(\mathbf{y}(t), t)$ for all $t \in[\tilde{t}, T]$. Following the same calculation as we did before, we have for $t \in[\tilde{t}, T]$,

$$
\begin{aligned}
|\dot{\mathbf{y}}(t)|^{2}-v^{2}(\mathbf{y}(t), t) & =v^{2}(\mathbf{x}, t)-v^{2}(\mathbf{y}, t)+\varepsilon^{2}+2 \varepsilon v(\mathbf{x}, t) \mathbf{n}(t) \cdot \mathbf{e} \\
& \leqslant 2 v(\mathbf{x}, t) \varepsilon\left\{\frac{\kappa^{\alpha}|\mathbf{x}-\mathbf{y}|^{\alpha}}{\varepsilon}+\frac{\varepsilon}{2 v(\mathbf{x}, t)}+\mathbf{e} \cdot \mathbf{n}(t)\right\} \\
& \leqslant 2 v(\mathbf{x}, t) \varepsilon\left\{\frac{\kappa^{\alpha} L^{\alpha}}{\varepsilon}+\frac{\varepsilon}{2}+\mathbf{e} \cdot \mathbf{n}(t)\right\} \\
& \leqslant 2 v(\mathbf{x}, t) \varepsilon\left\{\frac{2}{3} \sin \frac{\theta_{0}}{2}+\mathbf{e} \cdot \mathbf{n}(t)\right\} .
\end{aligned}
$$


(i) Suppose $T \geqslant t_{1}$. Then $\mathbf{n}_{0}(q)=\mathbf{n}(T)$ so that

$$
\begin{aligned}
\mathbf{e} \cdot \mathbf{n}(t) & =\mathbf{e} \cdot \mathbf{n}_{0}(q)+\mathbf{e} \cdot(\mathbf{n}(t)-\mathbf{n}(T)) \\
& \leqslant-\cos \frac{\theta_{0}}{2}+M \kappa^{\beta}(T-\tilde{t})^{\beta} \leqslant-\sin \frac{\theta_{0}}{2}+M\left(\frac{\kappa L}{\varepsilon}\right)^{\beta} \leqslant-\frac{2}{3} \sin \frac{\theta_{0}}{2} .
\end{aligned}
$$

(ii) Next suppose $T \in\left(0, t_{1}\right]$. Then $\mathbf{n}_{0}(q)=\mathbf{n}_{0}(p)$ and

$$
\begin{aligned}
\mathbf{e} \cdot \mathbf{n}(t) & =\mathbf{e} \cdot \mathbf{n}_{0}(p)+\mathbf{e} \cdot(\mathbf{n}(t)-\mathbf{n}(0)) \leqslant \mathbf{e} \cdot \mathbf{n}_{0}(p)+M \kappa^{\beta} t_{1}^{\beta} \\
& =\mathbf{e} \cdot \mathbf{n}(0)+M\left(\frac{\kappa L}{\varepsilon}\right)^{\beta} \leqslant \mathbf{e} \cdot \mathbf{n}(0)+\frac{1}{3} \sin \frac{\theta_{0}}{2} .
\end{aligned}
$$

Now decompose e as

$$
\mathbf{e}=-\cos \theta \mathbf{n}_{0}(p)+\sin \theta \mathbf{n}_{0}^{\perp}
$$

where $\theta \in[0, \pi]$ and $\mathbf{n}_{0}^{\perp}$ is a unit vector that is perpendicular to $\mathbf{n}_{0}(p)$. Then

$$
\cos \theta=-\mathbf{e} \cdot \mathbf{n}_{0}(p) \geqslant \cos \frac{\theta_{0}}{2}
$$

so $\theta \in\left(0, \theta_{0} / 2\right]$. Also,

$$
\left|\mathbf{n}(0) \cdot \mathbf{n}_{0}^{\perp}\right| \leqslant \sqrt{1-\left|\mathbf{n}(0) \cdot \mathbf{n}_{0}(p)\right|^{2}} \leqslant \sqrt{1-\sin ^{2} \theta_{0}}=\cos \theta_{0} .
$$

Hence,

$$
\begin{aligned}
-\mathbf{e} \cdot \mathbf{n}(0) & =\cos \theta \mathbf{n}_{0}(p) \cdot \mathbf{n}(0)-\sin \theta \mathbf{n}_{0}^{\perp} \cdot \mathbf{n}(0) \\
& \geqslant \cos \theta \sin \theta_{0}-\sin \theta \cos \theta_{0}=\sin \left(\theta_{0}-\theta\right) \geqslant \sin \frac{\theta_{0}}{2} .
\end{aligned}
$$

This implies that

$$
\mathbf{e} \cdot \mathbf{n}(t) \leqslant-\frac{2}{3} \sin \frac{\theta_{0}}{2}
$$

Thus, in any case (i) or (ii), we have $|\dot{\mathbf{y}}(t)| \leqslant v(\mathbf{y}(t), t)$ for all $t \in[\tilde{t}, T]$. This completes the proof.

Proof of Theorem 5. The proof is analogous to the locally estimate, with the modification that $\Gamma(t) \cap B\left(q, R_{1}\right)$ is divided into more than $2 n$ sets but the size $R_{1}$ of the ball is independent of $t$.

Let $\left\{\mathbf{e}_{i}\right\}_{i=1}^{N}$ be a minimum collection of unit vectors such that

$$
\mathbb{S}^{n-1} \subset \bigcup_{i=1}^{N} B\left(\mathbf{e}_{i}, 2 \sin \frac{\theta_{0}}{8}\right), \quad \mathbf{e}_{i} \in \mathbb{S}^{n-1} \quad \forall i=1, \cdots, N .
$$

One can show that $N \leqslant\left[32 \pi / \theta_{0}\right]^{n-1}$. Given $t>0$ we define

$$
\Gamma_{i}(t):=\left\{q \in \Gamma(t)|| \mathbf{n}_{0}(q)-\mathbf{e}_{i} \mid \leqslant 2 \sin \frac{\theta_{0}}{8}\right\} .
$$


Then

$$
\Gamma(t)=\bigcup_{i=1}^{N} \Gamma_{i}(t) .
$$

Fix any $q \in \Gamma(t)$. We show that each $\Gamma_{i}(t) \cap B\left(q, R_{1}\right)$ lies on a Lipschitz graph. For this, it is sufficient to consider $\Gamma_{1} \cap B\left(q, R_{1}\right)$. By rotation, we can assume that $\mathbf{e}_{1}=(1,0, \cdots, 0)$.

For every $p=\left(p_{1}, p^{\prime}\right) \in \Gamma_{1} \cap B\left(p, R_{1}\right)$, we define

$$
\varphi\left(p ; x^{\prime}\right)=p_{1}-\left|x^{\prime}-p^{\prime}\right| \cot \frac{\theta_{0}}{4} \quad \forall x^{\prime} \in \mathbb{R}^{n-1} .
$$

We claim that

$$
\left\{\left(x_{1}, x^{\prime}\right) \in B\left(\mathbf{0}, R_{1}\right) \mid x_{1} \leqslant \varphi\left(p ; x^{\prime}\right)\right\} \subset \mathrm{D}(t) .
$$

To this end, let $x$ be an arbitrary point in the set. Then $x \in B\left(p, 2 R_{1}\right)$. Set

$$
\mathbf{e}=(x-p) /|(x-p)| .
$$

The angle between $\mathbf{e}$ and $-\mathbf{e}_{1}$ is no more than $\theta_{0} / 4$. As the angle between $\mathbf{e}_{1}$ and $\mathbf{n}_{0}(q)$ is less that $\theta_{0} / 4$, we see that the angle between $\mathbf{e}$ and $-\mathbf{n}_{0}(q)$ is no more than $\theta_{0} / 2$. Hence, by Lemma 5.1, $x \in \mathrm{D}(t)$. This proves the claim.

Now define

$$
\psi_{1}\left(x^{\prime}\right)=\max _{p \in \Gamma_{i}(t)} \varphi\left(p ; x^{\prime}\right), \quad \forall x^{\prime} \in \mathbb{R}^{n-1} .
$$

Then $\psi_{1}$ is a Lipschitz function with Lipschitz constant no more than $\cot \frac{\theta_{0}}{4}$. In addition, for any $p=\left(x_{1}, x^{\prime}\right) \in \Gamma_{1}(t) \cap B(q, L / 2)$, we must have $x_{1}=\varphi\left(p ; x^{\prime}\right)=\psi_{1}\left(x^{\prime}\right)$. Hence,

$$
\begin{aligned}
\Gamma_{1}(t) \cap B\left(q, R_{1}\right) & \subset\left\{\left(x_{1}, x^{\prime}\right) \mid x_{1}=\psi\left(x^{\prime}\right)\right\}, \\
\left\{\left(x_{1}, x^{\prime}\right)\right. & \left.\in B\left(q, R_{1}\right) \mid x_{1} \leqslant \psi\left(x^{\prime}\right)\right\} \subset \mathrm{D}(t) .
\end{aligned}
$$

Similarly, we can define $\psi_{i}$ for $\Gamma_{i}(t) \cap B(p, L / 2)$ for each $i=2, \cdots, N$. This completes the proof.

\subsection{Lipschitz initial boundary}

Although not needed in the subsequent application, it is still interesting to investigate the regularity of the fronts when $\mathrm{D}_{0}$ has Lipschitz boundary.

Regarding the interior cone condition (5.1), one can show that if $p \in \Gamma_{0}$ and $\mathbf{n}_{0}(p) \cdot \mathbf{n}_{0}(q) \geqslant 0$ for all $q \in B(p, \rho)$ for some $\rho>0$, then $\Gamma_{0} \cap B(p, \rho)$ is a Lipschitz graph; namely, up to a rotation $\Gamma_{0} \cap B(p, \rho)$ is a graph in form $x_{1}=\psi_{0}\left(x_{2}, \cdots, x_{n}\right)$ where $\psi_{0}$ is a Lipschitz function.

If in addition $\mathbf{n}_{0}: \Gamma_{0} \rightarrow \mathbb{S}^{n-1}$ is a continuous function, then locally $\Gamma_{0}$ is a $C^{1}$ graph.

Here we investigate for small time the regularity of the front when initially it is Lipschitz.

Proposition 5.1 Suppose $\Gamma_{0}$ is Lipschitz. Then $\Gamma_{t}$ is also Lipschitz for every sufficiently small $t>0$. More precisely, locally $\Gamma(t)$ can be represented as, after a rotation, $x_{1}=h\left(x^{\prime}, t\right)$ for every small $t>0$. 
Proof. (i) Pick an arbitrarily point on $\Gamma_{0}$. By rotation and translation, we assume that this point is the origin $\mathbf{0}$ and

$$
\mathrm{D}_{0} \cap C\left(\mathbf{0}, 3 \delta_{0}\right)=\left\{\left(x_{1}, x^{\prime}\right) \in C\left(\mathbf{0}, 3 \delta_{0}\right) \mid x_{1} \leqslant \psi_{0}\left(x^{\prime}\right)\right\}
$$

where $\psi_{0}$ is Lipschitz with a constant bounded by, say, $\cot \theta_{0}$ with $\theta_{0} \in(0, \pi / 4)$.

Set $\delta_{1}=\delta_{0} \tan \theta_{0}$ so that $\left|\psi\left(x^{\prime}\right)\right| \leqslant \delta_{1} \cot \theta_{0}=\delta_{0}$ for every

$$
x^{\prime} \in B^{\prime}\left(\mathbf{0}^{\prime}, \delta_{1}\right):=\left\{x^{\prime} \in \mathbb{R}^{n-1}|| x^{\prime} \mid \leqslant \delta_{1}\right\} .
$$

Set $t_{1}=\delta_{0} \sin \theta_{0} / c$ so that

$$
\operatorname{dist}\left(\Gamma_{t}, \Gamma_{0}\right) \leqslant c t \leqslant \delta_{0} \sin \theta_{0}
$$

for every $t \in\left(0, t_{1}\right]$.

For each $x^{\prime} \in B^{\prime}\left(\mathbf{0}^{\prime}, \delta_{1}\right)$, the function $h \in \mathbb{R} \rightarrow \tau\left(h, x^{\prime}\right)$ is a continuous function satisfying $\tau\left(\psi_{0}\left(x^{\prime}\right), x^{\prime}\right)=0$ and $\tau\left(\psi_{0}\left(x^{\prime}\right)+c t / \sin \theta_{0}, x^{\prime}\right) \geqslant t$ for every $t \in\left(0, t_{1}\right]$. Hence,

$$
\begin{aligned}
h\left(x^{\prime}, t\right): & =\min \left\{h>\psi_{0}\left(x^{\prime}\right) \mid \tau\left(h, x^{\prime}\right)=t\right\} \\
& \in\left(\psi_{0}\left(x^{\prime}\right), \psi_{0}\left(x^{\prime}\right)+\frac{c t}{\sin \theta_{0}}\right] \subset\left[-\delta_{0}, 2 \delta_{0}\right] .
\end{aligned}
$$

By the continuity of $\tau(\cdot), \tau\left(h\left(x^{\prime}\right), x^{\prime}\right)=t$ for all $x^{\prime} \in B^{\prime}\left(\mathbf{0}^{\prime}, \delta_{1}\right)$ and $t \in\left(0, t_{1}\right]$. We shall show that $h(\cdot, t)$ is Lipschitz and locally $\Gamma(t)$ is a graph represented by $x_{1}=h\left(x^{\prime}, t\right)$.

(ii) Let $t \in\left(0, t_{1}\right]$ and

$$
q=\left(q_{1}, q^{\prime}\right) \in \Gamma(t) \cap C\left(\mathbf{0}, 2 \delta_{0}\right)
$$

be arbitrary. Let $\mathbf{x} \in \mathcal{C}_{b}(t)$ be such that $\mathbf{x}(t)=q$. Then

$$
\mathbf{x}(0) \in B(q, c t) \subset C\left(\mathbf{0}, 3 \delta_{0}\right) .
$$

Denote $\mathbf{n}(t)=\dot{\mathbf{x}}(t) /|\dot{\mathbf{x}}(t)|$. Then in terms of Theorem 3, we can derive that $\mathbf{e}_{1} \cdot \dot{\mathbf{n}}(0) \geqslant \sin \theta_{0}$. Consequently, as $|\mathbf{n}(t)-\mathbf{n}(0)| \leqslant M \kappa^{\beta} t_{1}^{\beta}$, we have $\mathbf{n}(t) \cdot \mathbf{e}_{1}>\frac{1}{2} \sin \theta_{0}$, by taking, if necessary, smaller $\delta_{0}$. This argument show that for every $t \in\left(0, t_{1}\right]$,

$$
\left\{q \in C\left(\mathbf{0}, 2 \delta_{0}\right) \cap \Gamma(t) \mid \mathbf{n} \cdot \mathbf{e}_{1} \geqslant \frac{\sin \theta_{0}}{2} \forall \mathbf{n} \in \mathbf{N}(q)\right\}=\Gamma(t) \cap C\left(\mathbf{0}, 2 \delta_{0}\right) .
$$

Now following the same proof as that in the local estimate, with $1 / \sqrt{n}$ being replaced by $\sin \theta_{0} / 2$ and the function $\psi\left(q ; x^{\prime}\right)=q_{1}-2 \sqrt{n}\left|x^{\prime}-q^{\prime}\right|$ being replaced by

$$
\psi\left(q ; x^{\prime}\right)=q_{1}-\frac{4}{\sin \theta_{0}}\left|q^{\prime}-x^{\prime}\right|, \quad \forall x^{\prime} \in \mathbb{R}^{n-1},
$$

we can conclude that $\Gamma(t) \cap C(q, \varepsilon)$ is contained in a graph $\left\{\left(x_{1}, x^{\prime}\right) \mid x_{1}=\psi\left(x^{\prime}\right)\right\}$ where $\varepsilon=$ $\varepsilon(t)$ is a small positive constant independent of $q$ and $\psi$ is a $q$ dependent Lipschitz function with Lipschitz constant no more than $4 / \sin \theta_{0}$.

(iii) Now for each $t \in\left(0, t_{1}\right]$ and each $z^{\prime} \in B^{\prime}\left(\mathbf{0}^{\prime}, \delta_{1}\right)$, applying the conclusion from (ii) at point $q=$ $\left(h^{\prime}\left(z^{\prime}, t\right), z^{\prime}\right)$ we conclude that $h^{\prime}(\cdot, t)$ is Lipschitz continuous in $B^{\prime}\left(z^{\prime}, \varepsilon\right)$ with Lipschitz constant no more than $4 / \sin \theta_{0}$. Consequently, $h(\cdot, t)$ is a Lipschitz continuous function on $B^{\prime}\left(\boldsymbol{0}^{\prime}, \delta_{1}\right)$. 
Finally, take $\delta_{2}=\delta_{1} / 2$ and $t_{2}=t_{1} / 4$. Let $t \in\left(0, t_{2}\right]$ and

$$
\left(z_{1}, z^{\prime}\right) \in \Gamma(t) \cap\left(\left[-\delta_{0}, 2 \delta_{0}\right] \times B^{\prime}\left(\mathbf{0}^{\prime}, \delta_{2}\right)\right) .
$$

We claim that $z_{1}=h\left(z^{\prime}, t\right)$. Suppose otherwise. Then $z_{1}>h\left(z^{\prime}, t\right)$ by the definition of $h$. Let $\mathbf{x} \in \mathrm{C}_{b}(t)$ be such that $\mathbf{x}(t)=\left(z_{1}, z^{\prime}\right)$. Then the trajectory $\mathbf{x}([0, t])$ is contained in

$$
\left[-\delta_{0}, 2 \delta_{0}\right] \times B^{\prime}\left(\mathbf{0}^{\prime}, \delta_{2}+c t\right) \subset\left[-\delta_{0}, 2 \delta_{0}\right] \times B^{\prime}\left(\mathbf{0}^{\prime}, \delta_{1}\right)
$$

Hence, it must intersect the surface

$$
\left\{\left(h\left(x^{\prime}, t\right), x^{\prime}\right) \mid x^{\prime} \in B^{\prime}\left(\mathbf{0}^{\prime}, \delta_{1}\right)\right\},
$$

i.e., $\mathbf{x}(\hat{t})=\left(h\left(x^{\prime}, t\right), x^{\prime}\right)$ at some time $\hat{t} \in(0, t)$ and some $x^{\prime} \in B^{\prime}\left(\mathbf{0}^{\prime}, \delta_{1}\right)$. But this is impossible since the arriving time to $\left(h\left(x^{\prime}, t\right), x^{\prime}\right)$ is exactly $t$.

In conclusion, for every $t \in\left[0, t_{2}\right], \Gamma(t) \cap\left(\left[-\delta_{0}, 2 \delta_{0}\right] \times B^{\prime}\left(\mathbf{0}^{\prime}, \delta_{2}\right)\right)$ is given by the graph

$$
x_{1}=h\left(x^{\prime}, t\right), \quad x^{\prime} \in B^{\prime}\left(\mathbf{0}^{\prime}, \delta_{2}\right) .
$$

This completes the proof.

\subsection{The area of the front $\Gamma(t)$}

In many applications, it is desirable to estimate the area of the front $\Gamma(t)$ in small balls. For this, we recall the area formula. Given a Lipschitz graph $S=\left\{\left(x_{1}, \psi\left(x^{\prime}\right) \mid x^{\prime} \in \Omega \subset \mathbb{R}^{n-1}\right\}\right.$, its area, denoted by $\mathfrak{H}^{n-1}(S)$, is given by

$$
\mathcal{H}^{n-1}(S)=\int_{\Omega} \sqrt{1+\mid \nabla_{x^{\prime}} \psi\left(x^{\prime 2}\right.} d x^{\prime}
$$

We immediately obtain the following:

Theorem 6 Assume (2.1) and (3.1). There exists $C(n)$ such that for every $t>0$ and $q \in \mathbb{R}^{n}$,

$$
\mathcal{H}^{n-1}(\Gamma(t) \cap B(q, \rho)) \leqslant C(n) \rho^{n-1}, \quad \forall 0<\rho \leqslant \min \left\{\frac{\kappa^{\beta} t^{1+\beta}}{2 \sqrt{n}}, \frac{R_{0}(n, c, \alpha)}{\kappa}\right\} .
$$

If also (5.1) holds, then there exists a constant $C\left(n, \theta_{0}\right)$ such that for every $t \geqslant 0$ and $q \in \mathbb{R}^{n}$,

$$
\mathcal{H}^{n-1}(\Gamma(t) \cap B(q, \rho)) \leqslant C\left(n, \theta_{0}\right) \rho^{n-1}, \quad \forall 0<\rho \leqslant \min \left\{\frac{\delta_{0}}{2}, \frac{R_{2}\left(n, c, \alpha, \theta_{0}\right)}{\kappa}\right\} .
$$

Proof. The assertion follows directly from the area formula and our earlier conclusion that locally $\Gamma(t)$ is contained in a union of finitely many Lipschitz graphs.

We remark that (1.2) for $\kappa \geqslant 1$ is a consequence of (5.2) since the ball of radius $1 / \kappa$ can be covered by at most $\left(2 / R_{2}\right)^{n}$ numbers of balls of radius $R_{2} / \kappa$. 


\section{Continuous dependence}

When $v$ is Lipschitz continuous in the space variable, solutions to the domain expansion problem are stable; i.e. if $v_{j} \rightarrow v$ and $\mathrm{D}_{0 j} \rightarrow \mathrm{D}_{0}$ as $j \rightarrow \infty$, then solutions of the domain expansion with speed $v_{j}$ starting from $\mathrm{D}_{j}$ approach that with speed $v$ starting from $\mathrm{D}_{0}$ as $j \rightarrow \infty$. When $v$ is not Lipschitz continuous, this stability may not hold; see Example 3 at the end of Section 2.

For the purpose of later application, we do need a certain kind of stability result. It happens that the function in our consideration has the following special properties:

$$
|\nabla v(x, t)| \leqslant K\{1+|\ln \operatorname{dist}(x, \mathrm{D}(t))|\}, \quad \forall x \notin \mathrm{D}(t), t \in[0, T]
$$

where $[0, T]$ is a time interval of consideration. It may seem strange at a first sight that the above property depends on the solution $Q=\cup_{0 \leqslant t \leqslant T}(\mathrm{D}(t) \times\{t\})$ of the domain expansion problem starting from $\mathrm{D}_{0}$; nevertheless, in studying free boundary problems, we quite often come up with such kind of estimates.

Without association with free boundary problems, we consider in general a function class that satisfies

$$
\begin{cases}|v(x, t)-v(y, t)| \leqslant \omega(|x-y|), \quad \forall x, y \in \mathbb{R}^{n}, & t \in[0, T], \\ \omega \in C^{1}((0, \infty) ;(0, \infty)), \quad \omega^{\prime} \geqslant 0 & \text { on }(0, \infty), \quad \int_{0}^{1} \frac{d h}{\omega(h)}=\infty .\end{cases}
$$

Here $\omega(\cdot)$ is quite often called the modulus of continuity. An example is $\omega(h)=h(1+|\ln h|)$.

Theorem 7 Let $\left(f, D_{0}\right)$ satisfy (2.1) and $Q=\cup_{t \in[0, T]}(\mathrm{D}(t) \times\{t\})$ be the solution of domain expansion with speed $v$ starting from $\mathrm{D}_{0}$. Assume either (6.1) or (6.2) holds.

Let $t \in(0, T], x \notin \mathrm{D}(t)$ and $\mathbf{x} \in \operatorname{Lip}\left([0, \mathrm{t}] ; \mathbb{R}^{\mathrm{n}}\right)$ satisfy $\mathbf{x}(t)=x$ and $|\dot{\mathbf{x}}(s)| \leqslant v(\mathbf{x}(s), s)$ for a.e. $s \in[0, t]$. Denote $d(s)=\operatorname{dist}(\mathbf{x}(s), \mathrm{D}(s))$. Then, with $\omega$ as in (6.2) or

$$
\omega(h)=2 K h(1+|\ln h|),
$$

we have

$$
\int_{d(s)}^{d(t)} \frac{d h}{\omega(h)} \leqslant t, \quad \forall s \in[0, t] .
$$

The above estimates shows that if $\mathbf{x}(t)$ is away from $\mathrm{D}(t)$, then $\mathbf{x}(s)$ is away from $\mathrm{D}(s)$ in a uniform manner. The implication of such a result, although not at all clear at this moment, will be seen clearly in the proof of next theorem. Indeed, as one will see in the later sections in the study of a free boundary problem, such a result is so vital that without it, one can hardly establish the existence of a meaningful solution of the underlying free boundary problem.

To cooperate (6.1) into the scope of (6.2), we first establish the following:

Lemma 6.1 Assume (6.1). Suppose $\rho:=\operatorname{dist}(x, \mathrm{D}(t))>0$. Then

$$
|v(x, t)-v(y, t)| \leqslant 2 K|x-y|\{1+|\ln \rho|\}, \quad \forall y \in \bar{B}(x, \rho) .
$$

Proof. Let $y \in \bar{B}(x, \rho)$. Set

$$
z(\theta)=y+\theta(x-y)
$$


for $\theta \in[0,1]$. Then

$$
\begin{aligned}
\operatorname{dist}(z(\theta), \mathrm{D}(t)) & \geqslant \operatorname{dist}(x, \mathrm{D}(t))-|x-z| \\
& =\rho-(1-\theta)|x-y| \geqslant \theta \rho .
\end{aligned}
$$

It then follows that

$$
\begin{aligned}
|v(x, t)-v(y, t)| & =\left|\int_{0}^{1} \frac{d}{d \theta} v(z(\theta), t) d \theta\right| \\
& =\left|\int_{0}^{1}(x-y) \cdot \nabla v(z(\theta), t) d \theta\right| \\
& \leqslant|x-y| \int_{0}^{1} K[1+|\ln (\theta \rho)|] d \theta \\
& \leqslant K|x-y| \int_{0}^{1}\{1+|\ln \rho|+|\ln \theta|\} d \theta \\
& =K|x-y|\{2+|\ln \rho|\} \\
& \leqslant 2 K|x-y|(1+|\ln \rho|) .
\end{aligned}
$$

Proof of Theorem 7. Clearly $d(\cdot)$ is a continuous function. Also $d(s)>0$ for all $s \in[0, t]$ since $\mathbf{x}(s) \in \mathrm{D}(s)$ for some $s \in[0, t)$ would imply $\mathbf{x}(t) \in \mathrm{D}(t)$. Set $\mathbf{e}(s)=\dot{\mathbf{x}}(s) / v(\mathbf{x}(s), s)$. Then

$$
\dot{\mathbf{x}}(s)=\mathbf{e}(s) v(\mathbf{x}(s), s), \quad|\mathbf{e}(s)| \leqslant 1, \quad \forall s \in[0, t] .
$$

Fix any $0 \leqslant t_{1}<t_{2} \leqslant t$. For a large integer $N$, set $h=\left(t_{2}-t_{1}\right) / N$ and $s_{i}=t_{1}+i h$. Fix an $i \in\{0,1, \cdots, N-1\}$. Let $y_{i} \in \Gamma\left(s_{i}\right)$ be a point such that

$$
d\left(s_{i}\right):=\operatorname{dist}\left(x\left(s_{i}\right), \mathrm{D}\left(s_{i}\right)\right)=\left|\mathbf{x}\left(s_{i}\right)-y_{i}\right| .
$$

Let $\mathbf{y}$ be (any one of the) solution to

$$
\dot{\mathbf{y}}(s)=\mathbf{e}(s) v(\mathbf{y}(s), s), \quad \forall s \in\left[s_{i}, s_{i+1}\right], \quad \mathbf{y}\left(s_{i}\right)=y_{i} .
$$

Then $\mathbf{y}\left(s_{i+1}\right) \in D\left(s_{i+1}\right)$ so that $d\left(s_{i+1}\right) \leqslant\left|\mathbf{y}\left(s_{i+1}\right)-\mathbf{x}\left(s_{i+1}\right)\right|$. To estimate $\left|\mathbf{y}\left(s_{i+1}\right)-\mathbf{x}\left(s_{i+1}\right)\right|$, we calculate, for $s \in\left(s_{i}, s_{i+1}\right)$,

$$
\begin{aligned}
\frac{d}{d s} \mid \mathbf{x}(s) & -\mathbf{y}(s) \mid=\frac{(\mathbf{x}-\mathbf{y}) \cdot \mathbf{e}}{|\mathbf{x}-\mathbf{y}|}(v(\mathbf{x}, s)-v(\mathbf{y}, s)) \\
& \leqslant|v(\mathbf{x}, s)-v(\mathbf{y}, s)| \\
& \leqslant\left|v\left(\mathbf{x}\left(s_{i}\right), s_{i}\right)-v\left(y_{i}, s_{i}\right)\right|+\left|v(\mathbf{x}, s)-v\left(\mathbf{x}\left(s_{i}\right), s_{i}\right)\right|+\left|v(\mathbf{y}, s)-v\left(y_{i}, s_{i}\right)\right| \\
& \leqslant \omega\left(\left|\mathbf{x}\left(s_{i}\right)-y_{i}\right|\right)+2 \varphi(h)
\end{aligned}
$$

by using either (6.2) or Lemma 6.1 in the case (6.1); here $\varphi(h)$ is defined by, for some fixed $R$ so large that $B(\mathbf{0}, R)$ covers all points in our consideration,

$$
\varphi(h)=\sup \left\{\left|v(z, s)-v\left(z^{\prime}, s^{\prime}\right)\right|: \begin{array}{l}
\left|s-s^{\prime}\right| \leqslant h,\left|z-z^{\prime}\right| \leqslant c h \\
s, s^{\prime} \in[0, T], z, z^{\prime} \in \bar{B}(\mathbf{0}, R)
\end{array}\right\} .
$$


By continuity we have $\varphi(h) \rightarrow 0$ as $h=1 / N \rightarrow 0$.Now integrating the differential inequality over $\left[s_{i}, s_{i+1}\right]$ and using $d\left(s_{i}\right)=\left|\mathbf{x}\left(s_{i}\right)-y_{i}\right|$ we have

$$
d\left(s_{i+1}\right) \leqslant\left|\mathbf{y}\left(s_{i+1}\right)-\mathbf{x}\left(s_{i+1}\right)\right| \leqslant d\left(s_{i}\right)+\int_{s_{i}}^{s_{i+1}}\left\{\omega\left(d\left(s_{i}\right)\right)+2 \varphi(h)\right\} d s .
$$

Consequently, adding these inequalities with $i=0, \cdots, N-1$ we obtain

$$
d\left(t_{2}\right) \leqslant d\left(t_{1}\right)+\sum_{i=0}^{N-1} \int_{s_{i}}^{s_{i+1}}\left\{\omega\left(d\left(s_{i}\right)\right)+2 \varphi(h)\right\} d s .
$$

Sending $N \rightarrow \infty$ and using the continuity of $d(\cdot)$ and the definition of Riemann integral we find that

$$
d\left(t_{2}\right) \leqslant d\left(t_{1}\right)+\int_{t_{1}}^{t_{2}} \omega(d(s)) d s, \quad \forall 0 \leqslant t_{1}<t_{2} \leqslant t .
$$

Now we can use a variation of the Gronwall's inequality to obtain our assertion. Introduce

$$
F(s):=d\left(t_{1}\right)+\int_{t_{1}}^{s} \omega\left(d\left(s^{\prime}\right)\right) d s^{\prime}, \quad \forall s \in\left[t_{1}, t\right] .
$$

Then $F\left(t_{1}\right)=d\left(t_{1}\right)$ and $d(s) \leqslant F(s)$ for all $s \in\left[t_{1}, t\right]$. Consequently,

$$
F^{\prime}(s)=\omega(d(s)) \leqslant \omega(F(s)), \quad \forall s \in\left[t_{1}, t\right]
$$

since $\omega(\cdot)$ is an increasing function. This implies that $F^{\prime} / \omega(F) \leqslant 1$, which gives, after integrating it over $\left[t_{1}, t\right]$,

$$
t-t_{1} \geqslant \int_{t_{1}}^{t} \frac{F^{\prime}(s) d s}{\omega(F(s))}=\int_{F\left(d_{1}\right)}^{F(t)} \frac{d h}{\omega(h)} \geqslant \int_{d\left(s_{1}\right)}^{d(t)} \frac{d h}{\omega(h)}
$$

since $d(t) \leqslant F(t)$. This completes the proof.

Now we come to the stability issue.

THEOREM 8 Let $\mathrm{D}_{0}$ be a bounded closed set and $\left\{v_{i}\right\}_{i=1}^{\infty}$ be a sequence of functions satisfying (2.1) with $v$ replaced by $v_{i}$. Let $Q_{i}=\cup_{0 \leqslant t \leqslant T}\left(\mathrm{D}_{i} \times\{t\}\right)$ be the solution to the domain expansion problem with speed $v_{i}$ starting from $\mathrm{D}_{0}$. Assume that one of the following holds:

(i) (6.2) is true with $v$ being replaced by $v_{i}$ for every $i \in \mathbb{N}$ (here $\omega$ is independent of $i$ );

(ii) (6.1) is true with $(v, D)$ being replaced by $\left(v_{i}, \mathrm{D}_{i}\right)$ for every $i \in \mathbb{N}(K$ is independent of $i)$.

Finally, assume that for some $v, \lim _{j \rightarrow \infty} v_{j}=v$ locally uniformly. Then

$$
\begin{aligned}
& \lim _{j \rightarrow \infty} \int_{0}^{T} \iint_{\mathbb{R}^{n}}\left|\chi_{Q_{j}}-\chi_{Q}\right| d x d t=0, \\
& \lim _{j \rightarrow \infty} \chi_{Q_{j}}(x, t)=\chi_{Q}(x, t), \quad \forall t \in[0, T], x \notin \Gamma(t):=\partial \mathrm{D}(t)
\end{aligned}
$$

where $Q=\cup_{0 \leqslant t \leqslant T}\{\mathrm{D}(t) \times\{t\}\}$ is the solution to domain expansion with speed $v$ starting from $\mathrm{D}_{0}$. 
Proof. Let $t \in(0, T]$ and $x \notin \Gamma(t)$ be fixed. We consider two cases

$$
\text { (i) } x \notin \mathrm{D}(t), \quad \text { (ii) } x \in \mathrm{D}(t) \backslash \Gamma(t) .
$$

(i) Suppose $x \notin \mathrm{D}(t)$. Then $\chi_{Q}(x, t)=0$. We claim that $\lim _{i \rightarrow \infty} \chi Q_{j}(x, t)=0$. Suppose not. Then there exists an integer sequence $\left\{i_{k}\right\}_{k=1}^{\infty}$ such that $\lim _{k \rightarrow \infty} i_{k}=\infty$ and $\chi Q_{i_{k}}(x, t)=1$ for all $k \in \mathbb{N}$. This implies that there exists $\left\{\mathbf{x}_{k}\right\}$ such that $\mathbf{x}_{k}(t)=x, \mathbf{x}_{k}(0) \in \mathrm{D}_{0}$ and

$$
\left|\mathbf{x}_{k}\left(t_{2}\right)-\mathbf{x}_{k}\left(t_{1}\right)\right| \leqslant \int_{t_{1}}^{t_{2}} v_{i_{k}}\left(\mathbf{x}_{k}(s), s\right) d s, \quad \forall 0 \leqslant t_{1}<t_{2} \leqslant t .
$$

It is easy to see that the family $\left\{\mathbf{x}_{k}\right\}$ is equicontinuous and bounded. Hence, along a subsequence, which we still denote by $\left\{\mathbf{x}_{k}\right\}$, it converges uniformly to a limit, say $\mathbf{x}$. Such a limit has the property that $\mathbf{x}(0) \in \mathrm{D}_{0}$ and $\mathbf{x}(t)=x$. In addition, as $v_{j} \rightarrow v$ locally uniformly as $j \rightarrow \infty$, we see that $\mathbf{x} \in \mathrm{C}(t)$. Hence, by the definition of the solution to domain expansion, $x \in \mathrm{D}(t)$, which contradicts the original assumption that $x \notin \mathrm{D}(t)$. Hence,

$$
\lim _{i \rightarrow \infty} \chi_{Q_{i}}(x, t)=\chi_{Q}(x, t)=0 .
$$

In this step, no regularity condition is needed. Now comes the hard part.

(ii) Suppose $x \in \mathrm{D}(t) \backslash \Gamma(t)$. Then $\chi_{Q}(x, t)=1$. We claim that $\chi_{Q_{j}}(x, t)=1$ for all sufficiently large $j$. We use a contradiction argument. Suppose this is not true. Then $\chi_{Q_{j}}(x, t)=0$ along a subsequence, which we still denote by $\left\{Q_{j}\right\}$ itself.

Let $t^{*}=\tau(x)$. As $x \notin \Gamma(t)$, we have $t^{*}<t$ so $\varepsilon:=t-t^{*}>0$. Let $\mathbf{x} \in \mathbb{C}_{b}\left(t^{*}\right)$ such that $\mathbf{x}\left(t^{*}\right)=x$. Denote $\mathbf{e}(s)=\dot{\mathbf{x}}(s) / v(\mathbf{x}(s), s)$. Now consider any fixed solution $\mathbf{y}_{j}$ to the differential equation

$$
\dot{\mathbf{y}}_{j}(s)=\mathbf{e}(s) v_{j}\left(\mathbf{y}_{j}(s)\right), \quad \forall s \in\left[0, t^{*}\right], \quad \mathbf{y}_{j}\left(t^{*}\right)=x .
$$

Such a family $\left\{\mathbf{y}_{j}\right\}$ is bounded and equicontinuous. Hence, along a subsequence, which we still denote by $\left\{\mathbf{y}_{j}\right\}$, it converges uniformly to a function $\mathbf{y}$. As $v_{j} \rightarrow v$ locally uniformly, we have

$$
\mathbf{y}(s)=\mathbf{e}(s) v(\mathbf{y}(s), s), \quad \forall s \in\left[0, t^{*}\right], \quad \mathbf{y}\left(t^{*}\right)=x .
$$

We now show that $\operatorname{dist}(\mathbf{y}(s), \Gamma(s))>0$ for all $s \in\left[0, t^{*}\right]$. To do this, we first note that $\chi Q_{j}(x, t)=0$ implies that $x \notin \mathrm{D}_{j}(t)$, i.e., $\tau_{j}(x)>t$. Hence,

$$
\begin{aligned}
& \operatorname{dist}\left(x, \Gamma_{j}\left(t^{*}\right)\right)=\min _{y \in \Gamma_{j}\left(t^{*}\right)}|x-y| \\
\geqslant & \min _{y \in \Gamma_{j}\left(t^{*}\right)}\left|\tau_{j}(x)-\tau_{j}(y)\right|=\tau_{j}(x)-t^{*}>t-t^{*}=\varepsilon .
\end{aligned}
$$

Now denoting $d_{j}(s)=\operatorname{dist}\left(\mathbf{y}_{j}(s), \mathrm{D}_{j}(s)\right)$, we have, by Theorem 6.1, for every $s \in\left[0, t^{*}\right]$,

$$
t \geqslant \int_{d_{j}(s)}^{d_{j}\left(t^{*}\right)} \frac{d h}{\omega(h)} \geqslant \int_{\operatorname{dist}\left(\mathbf{y}_{j}(s), \mathrm{D}_{j}(s)\right)}^{\varepsilon} \frac{d h}{\omega(h)} .
$$

Let $\delta \in(0, \varepsilon)$ be a constant such that $\int_{\delta}^{\varepsilon} \frac{d h}{\omega(h)}=t$. Then the above estimate gives us

$$
\operatorname{dist}\left(\mathbf{y}_{j}(s), \mathrm{D}_{j}(t)\right) \geqslant \delta, \quad \forall j \in \mathbb{N}, s \in\left[0, t^{*}\right] .
$$


As $\mathbf{y}_{j} \rightarrow \mathbf{y}$ uniformly, there exists $J$ such that $\left|\mathbf{y}_{j}(s)-\mathbf{y}(s)\right| \leqslant \delta / 2$ for all $j \geqslant J$ and all $s \in\left[0, t^{*}\right]$. Therefore,

$$
\operatorname{dist}\left(\mathbf{y}(s), \mathrm{D}_{j}(s)\right) \geqslant \delta / 2, \quad \forall j \geqslant J, s \in\left[0, t^{*}\right] .
$$

In particular,

$$
\operatorname{dist}\left(\mathbf{y}(0), \mathrm{D}_{0}\right)=\operatorname{dist}\left(\mathbf{y}(0), \mathrm{D}_{j}(0)\right) \geqslant \delta / 2
$$

Now we compare $\mathbf{x}$ and $\mathbf{y}$.Assume that (6.1) holds with $(v, \mathrm{D})$ being replaced by $\left(v_{j}, \mathrm{D}_{j}\right)$ for every $j \in \mathbb{N}$. This implies that for every $j \geqslant J$,

$$
\left|\nabla v_{j}(z, s)\right| \leqslant K[1+|\ln \delta / 4|], \quad \forall s \in\left[0, t^{*}\right], z \in B(\mathbf{y}(s), \delta / 4) .
$$

Now since $v_{j} \rightarrow v$ uniformly, we see that $v=\lim v_{j}$ share the same property:

$$
|\nabla v(z, s)| \leqslant K[1+|\ln \delta / 4|], \quad \forall s \in\left[0, t^{*}\right], z \in B(\mathbf{y}(s), \delta / 4) .
$$

Noting that both $\mathbf{x}$ and $\mathbf{y}$ satisfies the same equation on $\left[0, t^{*}\right]$ with same terminal value $\mathbf{x}\left(t^{*}\right)=$ $x=\mathbf{y}\left(t^{*}\right)$. The above Lipschitz continuity on $v$ then implies that $\mathbf{x}$ never diverges from $\mathbf{y}$, i.e. $\mathbf{x}(s) \equiv \mathbf{y}(s)$ for all $s \in\left[0, t^{*}\right]$. This contradicts $\mathbf{x}(0) \in \mathrm{D}_{0}$ and $\operatorname{dist}\left(\mathbf{y}(0), \mathrm{D}_{0}\right) \geqslant \delta / 2$.

Assume that (6.2) holds with $v$ being replaced by $v_{j}$ for every $j \in \mathbb{N}$. Then as $v_{j} \rightarrow v$ uniformly, we see that $v$ also satisfies (6.2). We can calculate

$$
-\frac{d}{d s}|\mathbf{x}(s)-\mathbf{y}(s)| \leqslant|v(\mathbf{x}, s)-v(\mathbf{y}, s)| \leqslant \omega(|\mathbf{x}(s)-\mathbf{y}(s)|), \quad \forall s \in\left[0, t^{*}\right] .
$$

Since $\left|\mathbf{x}\left(t^{*}\right)-\mathbf{y}\left(t^{*}\right)\right|=0$ and

$$
\int_{0}^{1} \frac{d h}{\omega(h)}=\infty
$$

we can derive that $|\mathbf{x}(s)-\mathbf{y}(s)|=0$ for all $s \in\left[0, t^{*}\right]$. Again, we have a contradiction.

The contradiction shows that $\chi Q_{j}(x, t)=1$ for all large $j$, so

$$
\lim _{j \rightarrow \infty} \chi_{Q_{j}}(x, t)=1=\chi_{Q}(x, t) .
$$

Finally, since the set $\cup_{t \in[0, T]}\{\Gamma(t) \times\{t\})$ is a Lipschitz graph $t=\tau(x)$ in the space-time domain, it has space-time measure zero. Hence, $\chi_{Q_{j}} \rightarrow \chi Q$ almost everywhere. The convergence of $\chi_{Q_{j}}-\chi_{Q}$ to zero in $L^{1}\left(\mathbb{R}^{n} \times[0, T]\right)$ then follows by the Lebesgue's Dominated Convergence theorem. This completes the proof.

REMARK 6.1 In Theorem 8, the condition (6.1) for $\left(v_{j}, D_{i}\right)$ can be replaced by

$$
\begin{cases}\left|\nabla v_{j}(x, t)\right| \leqslant K\left\{1+\left|\ln \operatorname{dist}\left(x, \mathrm{D}_{j}(t)\right)\right|\right\} & \text { if } \operatorname{dist}(x, \mathrm{D}(t))>1 / j, t \in[0, T], \\ \left|v_{j}(x, t)-v_{j}(y, t)\right| \leqslant K|x-y|^{\alpha}, & \forall x, y \in \mathbb{R}^{n}, t \in[0, T] .\end{cases}
$$

The revised conclusion of Lemma 6.1 is

$$
\left|v_{j}(x, t)-v_{j}(y, t)\right| \leqslant K j^{-\alpha}+2 K|x-y|[1+\ln \rho]
$$

where the extra term $K j^{-\alpha}$ comes from the thin layer of width $1 / j$ in which the derivative estimate is replaced by the Hölder estimate. Thus, Theorem 7 still holds if we replace $\omega$ by

$$
\omega_{j}(h)=K j^{-\alpha}+2 K h(1+|\ln h|) .
$$


The proof of Theorem 8 remains unchanged except we replace $\omega$ by $\omega_{j}$. Note that for every $\varepsilon>0$, there exists $\delta \in(0, \varepsilon)$ and an integer $J \gg 1$ such that

$$
\int_{\delta}^{\varepsilon} \frac{d h}{\omega_{j}(h)}>t, \quad \forall j \geqslant J .
$$

\section{A singular layer potential}

In this section we study the solution $w$ to the heat equation

$$
\begin{cases}\frac{\partial w}{\partial t}-\Delta w=\frac{\partial}{\partial t} \chi_{\mathrm{D}(t)} & \text { in } \mathbb{R}^{n} \times[0, \infty), \\ w(\cdot, 0)=0 & \text { on } \mathbb{R}^{n} \times\{0\}\end{cases}
$$

where

$$
Q=\cup_{t \geqslant 0}(\mathrm{D}(t) \times\{t\})
$$

is the solution to the domain expansion with speed $v$ starting from $\mathrm{D}_{0}$.

Due to the singular nature of the characteristic function, the equation is interpreted in the sense of distribution. Since the equation is linear, we can use the fundamental solution of the heat equation to express the solution in terms of a singular layer potential. We denote the fundamental solution by

$$
K(x, t):=(4 \pi t)^{-n / 2} e^{-|x|^{2} /(4 t)}, \quad \forall x \in \mathbb{R}^{n}, t>0 .
$$

Then the solution can be expressed as, for every $x \in \mathbb{R}^{n}$ and $t>0$,

$$
\begin{aligned}
w(x, t) & =\iint_{\mathbb{R}^{n}} \int_{0}^{t} K(x-y, t-s) \frac{\partial}{\partial s} \chi_{Q}(y, s) d s d y \\
& =\iint_{\mathrm{D}(t) \backslash \mathrm{D}(0)} K(x-y, t-\tau(y)) d y
\end{aligned}
$$

since $\partial_{s} \chi_{Q}$ is a Delta function with mass at $s=\tau(y)$. Note that $w$ is positive and

$$
\begin{aligned}
\iint_{\mathbb{R}^{n}} w(x, t) d x & =\iint_{\mathrm{D}(t) \backslash \mathrm{D}(0)} \iint_{\mathbb{R}^{n}} K(x-y, t-\tau(y)) d x d y \\
& =|\mathrm{D}(t) \backslash \mathrm{D}(0)|, \quad \forall t \geqslant 0 .
\end{aligned}
$$

Also, it is easy to see that $w$ is smooth in the region away from the lateral boundary of $Q$.

The main purpose of this section is to provide a bound on the spatial Hölder norm. In the sequel, $C(n, \alpha)$ denotes various constants that depend only on $n$ and $\alpha$, whereas $C(n)$ that depends only on $n$.

THEOREM 9 Let $\{\mathrm{D}(t)\}_{t \geqslant 0}$ be a collection of bounded closed sets satisfying the following:

$$
\begin{aligned}
& \mathrm{D}(s) \subset \mathrm{D}(t), \quad|t-s| \leqslant \operatorname{dist}(x, \mathrm{D}(s)) \leqslant c|t-s|, \quad \forall 0 \leqslant s \leqslant t, x \in \Gamma(t), \\
& \mathcal{H}^{n-1}(\Gamma(t) \cap B(x, \rho)) \leqslant C^{*} \rho^{n-1}, \quad \forall \rho \in\left(0, R_{*}\right], x \in \mathbb{R}^{n}, t \geqslant 0
\end{aligned}
$$


where $\Gamma(t):=\partial \mathrm{D}(t)$ and $c \geqslant 1, C^{*} \geqslant 1$ and $R_{*} \in(0,1]$ are constants. Then $w$ from (7.1) satisfies

$$
\begin{gathered}
\frac{\left|w\left(x_{1}, t\right)-w\left(x_{2}, t\right)\right|}{\left|x_{1}-x_{2}\right|^{\alpha}} \leqslant \frac{c C(n, \alpha) C^{*}}{R_{*}^{\alpha / 2}}, \quad \forall x_{1}, x_{2} \in \mathbb{R}^{n}, t \geqslant 0, \quad \alpha \in(0,1), \\
|\nabla w(x, t)| \leqslant c C(n) C^{*}\left[\frac{1}{\sqrt{R_{*}}}+\ln \frac{\sqrt{R_{*}}+\operatorname{dist}(x, \Gamma(t))}{\operatorname{dist}(x, \Gamma(t))}\right], \quad \forall t>0, x \notin \Gamma(t) .
\end{gathered}
$$

The estimate for $\alpha / 2$ Hölder norm in the $t$ variable is analogous and is omitted. An important feature of the bound is the factor $R_{*}^{\alpha / 2}$ instead of $R_{*}^{\alpha}$ which is relatively easy to obtain.

When $\rho>R_{*}$, we can cover $B(x, \rho)$ by $\left(2 \rho / R_{*}\right)^{n}$ many balls of radius $R_{*}$ to obtain the estimate

$$
\begin{aligned}
\mathcal{H}^{n-1}(\Gamma(t) \cap B(x, \rho)) & \leqslant\left(\frac{2 \rho}{R_{*}}\right)^{n} \sup _{y \in \mathbb{R}^{n}} \mathcal{H}^{n-1}\left(\Gamma(t) \cap B\left(y, R_{*}\right)\right) \\
& \leqslant \frac{2^{n} C^{*} \rho^{n}}{R_{*}}, \quad \forall x \in \mathbb{R}^{n}, \rho \geqslant R_{*} .
\end{aligned}
$$

Regularities for singular integral of heat kernel are related to the following singular kernel

$$
K_{\alpha}(x, t):=t^{-(n+\alpha) / 2} e^{-|x|^{2} /(8 t)}, \quad \alpha \geqslant 0 .
$$

LEMmA 7.1 There exists a constant $C(n)$ such that for every $x, x_{1}, x_{2} \in \mathbb{R}^{n}$,

$$
\begin{gathered}
|\nabla w(x, t)| \leqslant C(n) \iint_{\mathrm{D}(t) \backslash \mathrm{D}_{0}} K_{1}(x-y, t-\tau(y)) d y, \\
\frac{\left|u\left(x_{1}, t\right)-u\left(x_{2}, t\right)\right|}{\left|x_{1}-x_{2}\right|^{\alpha}} \leqslant C(n) \sup _{z \in \mathbb{R}^{n}} \iint_{\mathrm{D}(t) \backslash \mathrm{D}(0)} K_{\alpha}(z-y, t-\tau(y)) d y, \quad \forall \alpha \in[0,1) .
\end{gathered}
$$

Proof. Notice that for every $z \in \mathbb{R}^{n}, s>0$, and $\alpha \in[0,1]$,

$$
\begin{gathered}
s^{-\alpha / 2} K(z, s)=s^{-\alpha / 2}(4 \pi s)^{-n / 2} e^{-|z|^{2} /(4 s)} \leqslant(4 \pi)^{-n / 2} K_{\alpha}(z, s), \\
s^{(1-\alpha) / 2}|\nabla K(z, s)| \leqslant K_{\alpha}(z, s) \sup _{\rho \geqslant 0}\left(\frac{\rho e^{-\rho^{2} / 8}}{\pi^{n / 2} 2^{n+1}}\right) \leqslant \frac{2 e^{-1 / 2}}{\pi^{n / 2} 2^{n+1}} K_{\alpha}(z, s) .
\end{gathered}
$$

The assertion for $|\nabla w|$ thus follows from

$$
\nabla w(x, t)=\iint_{\mathrm{D}(t) \backslash \mathrm{D}(0)} \nabla K(x-y, t-\tau(y)) d y .
$$

Now we estimate the Hölder norm. From this, we can estimate, for every different $z_{1}$ and $z_{2}$ in $\mathbb{R}^{n}$,

$$
\begin{aligned}
\frac{\left|K\left(z_{1}, s\right)-K\left(z_{2}, s\right)\right|}{\left|z_{1}-z_{2}\right|^{\alpha}} & =\left|K\left(z_{1}, s\right)-K\left(z_{2}, s\right)\right|^{1-\alpha}\left|\int_{0}^{1} \frac{\left(z_{2}-z_{1}\right) \cdot \nabla K\left(z_{1}+\varsigma\left(z_{2}-z_{1}\right), s\right)}{\left|z_{1}-z_{2}\right|} d \varsigma\right|^{\alpha} \\
& \leqslant \frac{1-\alpha}{s^{\alpha / 2}}\left|K\left(z_{1}, s\right)-K\left(z_{2}, s\right)\right|+\alpha s^{(1-\alpha) / 2} \int_{0}^{1}\left|\nabla K\left(z_{1}+\varsigma\left(z_{2}-z_{1}\right), s\right)\right| d \varsigma \\
& \leqslant C(n)\left\{K_{\alpha}\left(z_{1}, s\right)+K_{\alpha}\left(z_{2}, s\right)+\int_{0}^{1} K_{\alpha}\left(z_{1}+\varsigma\left(z_{2}-z_{1}\right), s\right) d \varsigma\right\}
\end{aligned}
$$


where in the first inequality we have used

$$
a^{1-\alpha} b^{\alpha} \leqslant(1-\alpha) a+\alpha b
$$

for positive $a$ and $b$.

Applying the above estimate with $z_{1}=x_{1}-y, z_{2}=x_{2}-y$ and $s=t-\tau(y)$ we obtain

$$
\begin{aligned}
\frac{\left|w\left(x_{1}, t\right)-w\left(x_{2}, t\right)\right|}{\left|x_{1}-x_{2}\right|^{\alpha}} \leqslant & \iint_{\mathrm{D}(t) \backslash \mathrm{D}(0)} \frac{\left|K\left(x_{1}-y, t-\tau(y)\right)-K\left(x_{2}-y, t-\tau(y)\right)\right|}{\left|\left(x_{1}-y\right)-\left(x_{2}-y\right)\right|^{\alpha}} d y \\
& \leqslant C(n) \iint_{\mathrm{D}(t) \backslash \mathrm{D}(0)}\left\{K_{\alpha}\left(x_{1}-y, t-\tau(y)\right)+K_{\alpha}\left(x_{2}-y, t-\tau(y)\right)\right. \\
& \left.\quad+\int_{0}^{1} K_{\alpha}\left(x_{1}+\varsigma\left(x_{2}-x_{1}\right)-y, t-\tau(y)\right) d \varsigma\right\} d y \\
& \leqslant 3 C(n) \sup _{z \in \mathbb{R}^{n}} \iint_{\mathrm{D}(t) \backslash \mathrm{D}(0)} K_{\alpha}(z-y, t-\tau(y)) d y .
\end{aligned}
$$

This completes the proof.

Proof of Theorem 9. The case $n=1$ is easy since (i) for each $x \in \mathbb{R}$, there are at most two boundary characteristic trajectories that stop at $x$, and (ii) each boundary characteristic curve is monotonic with slope between 1 and $c$ (regarding $t$ as the horizontal axis). Indeed, the potential $w$ is Lipschitz continuous with a Lipschitz constant depending only on $c$ and the number of connected pieces of $\mathrm{D}_{0}$; we omit the details. Hence we consider the case $n \geqslant 2$ so the regularity of fronts plays an essential role.

Let $\alpha \in(0,1]$ and $(x, t) \in \mathbb{R}^{n} \times(0, \infty)$. The following division of three pieces is based on but significantly simplifies the original calculation by Su and Burger in [34]:

$$
\iint_{\mathrm{D}(t) \backslash \mathrm{D}(0)} K_{\alpha}(x-y, t-\tau(y)) d y=\left(\iint_{\Omega_{1}}+\iint_{\Omega_{2}}+\iint_{\Omega_{3}}\right) K_{\alpha}(x-y, t-\tau(y)) d y
$$

where

$$
\begin{aligned}
& \Omega_{1}:=\mathrm{D}(t) \backslash\left(B\left(x, \sqrt{R_{*}}\right) \cup \mathrm{D}(0)\right), \\
& \Omega_{2}:=\mathrm{D}(t) \cap B\left(x, \sqrt{R_{*}}\right) \backslash\left(B\left(x, R_{*}\right) \cup \mathrm{D}(0)\right), \\
& \Omega_{3}:=\mathrm{D}(t) \cap B\left(x, R_{*}\right) \backslash \mathrm{D}(0) .
\end{aligned}
$$

1. Contribution from far range. We estimate the integral of $K_{\alpha}$ on $\Omega_{1}$ by using

$$
K_{\alpha}(z, s)=|z|^{-n-\alpha}\left(\frac{|z|^{2}}{s}\right)^{(n+\alpha) / 2} e^{-|z|^{2} /(8 s)} \leqslant C(n)|z|^{-n-\alpha} .
$$

Hence,

$$
\iint_{\Omega_{1}} K_{\alpha}(x-y, t-\tau(y)) d y \leqslant C(n) \iint_{\mathbb{R}^{n} \backslash B\left(x, \sqrt{R_{*}}\right)}|x-y|^{-n-\alpha} d y \leqslant C(n, \alpha) R_{*}^{-\alpha / 2} .
$$


2. Contribution from intermediate range. To estimate the integral on $\Omega_{2}$, we use $d y=$ $\left.d \mathcal{H}^{n-1}\right|_{\Gamma(s)} v(y, s) d s$. Since

$$
t-s \leqslant \operatorname{dist}(x, \mathrm{D}(s)) \leqslant c(t-s)
$$

for every $x \in \Gamma(t)$ and $0 \leqslant s<t$, we have $1 \leqslant v \leqslant c$. Hence

$$
\begin{aligned}
\iint_{\Omega_{2}} K_{\alpha}(x-y, t-\tau(y)) d y & =\int_{0}^{t} \int_{\Gamma(s) \cap B\left(x, \sqrt{R_{*}}\right) \backslash B\left(x, R_{*}\right)} K_{\alpha}(x-y, t-s) v(y, s) d \mathcal{H}^{n-1} d s \\
& \leqslant c \int_{0}^{t} \int_{\Gamma(t-s) \cap B\left(x, \sqrt{R_{*}}\right) \backslash B\left(x, R_{*}\right)} K_{\alpha}(x-y, s) d \mathcal{H}^{n-1} d s .
\end{aligned}
$$

Let $J$ be an integer such that

$$
\sqrt{R^{*}} \in\left(2^{J-1} R_{*}, 2^{J} R_{*}\right]
$$

We define $\rho_{i}=2^{i} R_{*}$ for $i=0, \cdots, J$. Using

$$
\mathcal{H}^{n-1}(\Gamma(t-s) \cap B(x, \rho)) \leqslant C(n) C^{*} \rho^{n} / R_{*}
$$

for all $\rho \geqslant R_{*}$ we have

$$
\begin{aligned}
\int_{\Gamma(t-s) \cap B\left(x, \sqrt{R_{*}}\right) \backslash B\left(x, R_{*}\right)} & K_{\alpha}(x-y, s) d \mathcal{H}^{n-1} \\
& \leqslant \sum_{i=1}^{J} \int_{\Gamma(t-s) \cap B\left(x, \rho_{i}\right) \backslash B\left(x, \rho_{i-1}\right)} K_{\alpha}(x-y, s) d \mathcal{H}^{n-1} \\
& \leqslant s^{-(n+\alpha) / 2} \sum_{i=1}^{J} e^{-\rho_{i-1}^{2} /(8 s)} \mathcal{H}^{n-1}\left(\Gamma(t-s) \cap B\left(x, \rho_{i}\right)\right) \\
& \leqslant \frac{C(n) C^{*}}{R_{*} S^{(n+\alpha) / 2}} \sum_{i=1}^{J} \rho_{i}^{n} e^{-\rho_{i-1}^{2} /(8 s)} \\
& =\frac{2^{n} C(n) C^{*}}{R_{*} S^{(n+\alpha) / 2}} \sum_{i=1}^{J} \rho_{i-1}^{n-1} e^{-\rho_{i}^{2} /(32 s)}\left(\rho_{i}-\rho_{i-1}\right) \\
& \leqslant \frac{2^{n} C(n) C^{*}}{R_{*} S^{(n+\alpha) / 2}} \int_{R_{*}}^{2 \sqrt{R_{*}}} \rho^{n-1} e^{-\rho^{2} /(32 s)} d \rho .
\end{aligned}
$$

Hence, using the same $C(n)$ for different constants depending only on $n$, we have

$$
\begin{aligned}
\left.\iint_{\Omega_{2}} K_{\alpha}(x-y), s\right) d \mathcal{H}^{n-1} & \leqslant \frac{c C(n) C^{*}}{R_{*}} \int_{0}^{\infty} \int_{0}^{2 \sqrt{R_{*}}} \frac{\rho^{n-1} e^{-\rho^{2} /(32 s)}}{s^{(n+\alpha) / 2}} d \rho d s \\
& =c C^{*} C(n, \alpha) R_{*}^{-\alpha / 2} .
\end{aligned}
$$

3. Contribution from close range. Same as above, we have

$$
\iint_{\Omega_{3}} K_{\alpha}(x-y, t-\tau(y)) d y \leqslant c \int_{0}^{t} \int_{\Gamma(t-s) \cap B\left(x, R_{*}\right)} K_{\alpha}(x-y, s) d \mathcal{H}^{n-1} d s .
$$


We define

$$
h(s):=\operatorname{dist}(x, \Gamma(t-s)), \quad I:=\left\{s \in(0, t) \mid h(s)<R_{*}\right\} .
$$

Note that when $s \in(0, t)$,

$$
\begin{aligned}
h(s) & =\operatorname{dist}(x, \Gamma(t-s)) \\
& \geqslant \operatorname{dist}(\Gamma(\tau(x)), \Gamma(t-s)) \geqslant|t-\tau(x)-s| .
\end{aligned}
$$

There is at most one $s \in[0, t]$ such that $h=0$. As we are performing integration, such a point does not matter. Hence, we consider only the case $h(s)>0$. Clearly, $\Gamma(t-s) \cap B(x, h(s))=\emptyset$. Also, $\Gamma(t-s) \cap B\left(x, R_{*}\right)=\emptyset$ if $s \in(0, t) \backslash I$.

Consider $s \in I$. Let $J$ be an integer such that

$$
2^{J-1} h(s)<R_{*} \leqslant 2^{J} h(s) .
$$

We define $\rho_{i}=2^{i} h(s)$ for $i=0, \cdots, J-1$ and define $\rho_{J}:=R_{*}$. Using

$$
\mathcal{H}^{n-1}(\Gamma(t-s) \cap B(x, \rho)) \leqslant C^{*} \rho^{n-1}
$$

for all $\rho \leqslant R_{*}$ and a similar calculation as above we obtain

$$
\begin{aligned}
\int_{\Gamma(t-s) \cap B\left(x, R_{*}\right)} & K_{\alpha}(x-y, s) d \mathcal{H}^{n-1} \\
& \left.=\sum_{i=1}^{J} \int_{\Gamma(t-s) \cap B\left(x, \rho_{i}\right) \backslash B\left(x, \rho_{i}-1\right)} K_{\alpha}(x-y), s\right) d \mathcal{H}^{n-1} \\
& \leqslant 2^{n-1} C^{*} s^{-(n+\alpha) / 2} \int_{h(s)}^{2 R^{*}} \rho^{n-2} e^{-\rho^{2} /(32 s)} d \rho \\
& \leqslant 2^{n-1} C^{*} s^{-(n+\alpha) / 2} e^{-h^{2}(s) /(64 s)} \int_{0}^{\infty} \rho^{n-2} e^{-\rho^{2} /(64 s)} d \rho \\
& =C(n) C^{*} s^{-(1+\alpha) / 2} e^{-h^{2}(s) /(64 s)} .
\end{aligned}
$$

Now we consider two cases:

(i) $\quad \alpha \in(0,1)$. Since $h(s)<R_{*}$ implies that

$$
|t-\tau(x)-s|<R_{*},
$$

so that

$$
\begin{aligned}
\iint_{\Omega_{3}} K_{\alpha}(x-y, t-\tau(y)) d y & \leqslant c C^{*} C(n) \int_{s \in[0, t],|t-\tau(x)-s|<R_{*}} s^{-(1+\alpha) / 2} d s \\
& \leqslant c C^{*} C(n, \alpha) R_{*}^{(1-\alpha) / 2} .
\end{aligned}
$$

(ii) $\alpha=1$. We have

$$
\iint_{\Omega_{3}} K_{1}(x-y, t-\tau(y)) d y \leqslant c C(n) C^{*} \int_{I} \frac{e^{-h(s)^{2} /(64 s)}}{s} d s .
$$


Let $h_{0}=t-\tau(x)$. We consider three cases.

1. $h_{0} \leqslant-R^{*}$. Then

$$
h(s) \geqslant|t-\tau(x)-s| \geqslant R^{*}
$$

for all $s>0$ so $I=\emptyset$.

2. $h_{0}>2 R_{*}$. Then $I \subset\left[h_{0}-R_{*}, h_{0}+R_{*}\right]$ so that

$$
\int_{I} \frac{e^{-h^{2} /(64 s)}}{s} d s \leqslant \int_{h_{0}-R_{*}}^{h_{0}+R_{*}} \frac{d s}{s}=\ln \frac{h_{0}+R_{*}}{h_{0}-R_{*}} \leqslant \ln 3 .
$$

3. $h_{0} \in\left(-R_{*}, 2 R^{*}\right]$. Then $I \subset\left(0,3 R^{*}\right]$ and

$$
\frac{h^{2}(s)}{s} \geqslant \frac{\left(h_{0}-s\right)^{2}}{s}=\frac{h_{0}^{2}}{s}-2 h_{0}+s \geqslant \frac{h_{0}^{2}}{s}-2
$$

since $R_{*} \leqslant 1$. Hence

$$
\begin{aligned}
\int_{I} \frac{e^{-h^{2} /(64 s)}}{s} d s & \leqslant e^{1 / 32} \int_{0}^{3 R^{*}} \frac{e^{-h_{0}^{2} /(64 s)}}{s} d s=e^{1 / 32} \int_{h_{0}^{2} /\left(192 R^{*}\right)}^{\infty} \frac{e^{-\eta}}{\eta} d \eta \\
& \leqslant C+e^{1 / 32} \ln \frac{R_{*}}{h_{0}^{2}} \leqslant C+2 e^{1 / 32} \ln \frac{\sqrt{R_{*}}+h_{0}}{h_{0}} .
\end{aligned}
$$

In summary, noting that

$$
\operatorname{dist}(x, \Gamma(t)) \geqslant|t-\tau(x)|=h_{0}
$$

we have

$$
\iint_{\Omega_{3}} K_{1}(x-y, t-\tau(y)) d y \leqslant c C^{*} C(n)\left\{1+\ln \frac{\sqrt{R_{*}}+\operatorname{dist}(x, \Gamma(t))}{\operatorname{dist}(x, \Gamma(t))}\right\} .
$$

4. Now we combine all these estimates. When $\alpha \in(0,1)$,

$$
\begin{aligned}
\iint_{\mathrm{D}(t) \backslash \mathrm{D}(0)} K_{\alpha}(x-y, t-\tau(y)) d y & =\left(\iint_{\Omega_{1}}+\iint_{\Omega_{2}}+\iint_{\Omega_{3}}\right) K_{\alpha} d y \\
& \leqslant C(n, \alpha)\left\{R_{*}^{-\alpha / 2}+C^{*} R_{*}^{-\alpha / 2}+C^{*} R_{*}^{(1-\alpha) / 2}\right\} . \\
& \leqslant \frac{C(n, \alpha) C^{*}}{R_{*}^{\alpha / 2}} .
\end{aligned}
$$

Together with Lemma 7.1, we obtain the assertion of the theorem for the Hölder norm. Similarly, we can obtain the estimate for $|\nabla w(x, t)|$. This completes the proof of Theorem 9 .

\section{Solidification with kinetic undercooling}

Now we consider the following mathematical model for the solidification in undercooled liquid:

$$
\left\{\begin{array}{l}
\frac{\partial}{\partial t}\left(u-\chi_{Q}\right)=\Delta u-f \quad \text { in } \mathbb{R}^{n} \times(0, T], \\
Q=\left\{(\mathbf{x}(t), t)\left|t \in[0, T], \mathbf{x}(0) \in \mathrm{D}_{0},\right| \dot{\mathbf{x}}(s) \mid \leqslant V(u(\mathbf{x}(s), s)) \text { a.e. } s \in[0, t]\right\}, \\
u(\cdot, 0)=u_{0}(\cdot) \quad \text { on } \mathbb{R}^{n} \times\{0\} .
\end{array}\right.
$$


The problem here is to find unknown $(u, Q)$, for each given $\mathrm{D}_{0}, f, u_{0}$ and $T>0$. We assume that

$$
1 \leqslant V(\varsigma) \leqslant c, \quad\left|V\left(\varsigma_{1}\right)-V\left(\varsigma_{2}\right)\right| \leqslant L\left|\varsigma_{1}-\varsigma_{2}\right|, \quad \forall \varsigma_{\varsigma_{1}}, \varsigma_{2} \in \mathbb{R} .
$$

THEOREM 10 Assume that $D_{0}$ is a bounded closed set satisfying the interior cone condition, that $u_{0}: \mathbb{R}^{n} \rightarrow \mathbb{R}$ is bounded and $C^{1}$ and $f: \mathbb{R}^{n} \times[0, \infty) \rightarrow \mathbb{R}$ is bounded. Also assume that $V$ satisfies (8.2).

Then for every $T>0,(8.1)$ admits a solution $(u, Q)$ where $u \in C^{\gamma, \gamma / 2}\left(\mathbb{R}^{n} \times[0, T]\right)$ for every $\gamma \in(0,1)$ and for each $t \in(0, T], \Gamma(t)=\partial Q \cap\left(\mathbb{R}^{n} \times\{t\}\right)$ is contained in a union of finitely many Lipschitz graphs.

Proof. We shall use Schauder's fixed point theorem to establish the existence.

1. We denote by the same $u_{0}$ its extension from $C^{1}\left(\mathbb{R}^{n} \times\{0\}\right)$ to $C^{1,1 / 2}\left(\mathbb{R}^{n} \times[0, T]\right)$ according to

$$
u_{0 t}=\Delta u_{0}-f \quad \text { on } \mathbb{R}^{n} \times(0, T] .
$$

Let $d_{0}$ be a constant such that $D_{0} \subset B\left(\mathbf{0}, d_{0}\right)$. Set $d_{T}=d_{0}+c T$. Since we know that the propagation speed is at most $c$, a priori we known that $\mathrm{D}(T) \subset B\left(\mathbf{0}, d_{T}\right)$. Hence, only those values of $u$ in the set $B\left(\mathbf{0}, d_{T}\right) \times[0, T]$ are relevant to our motion problem. Thus we work on $\mathbf{X}:=$ $C\left(\bar{B}\left(\mathbf{0}, d_{T}\right) \times[0, T]\right)$. Let $\alpha \in(0,1)$ be fixed. For a constant $\kappa \geqslant 1$ to be determined, we work on the closed convex set

$$
\mathbf{X}(\kappa):=\left\{u \in \mathbf{X} \mid[u]_{\alpha}:=\sup _{t \in[0, T]} \sup _{x, y \in \bar{B}\left(\mathbf{0}, d_{T}\right), x \neq y} \frac{|u(x, t)-u(y, t)|}{|x-y|^{\alpha}} \leqslant \frac{\kappa^{\alpha}}{L}\right\} .
$$

2. Fix any $u \in \mathbf{X}(\kappa)$. Let $v=V \circ u$. Then $v$ is continuous and $1 \leqslant v \leqslant c$ and $[v]_{\alpha} \leqslant \kappa^{\alpha}$.

Taking $v$ as speed, we solve the problem of domain expansion starting from $D_{0}$, according to (1.1). As the maximum propagation speed is $c$, information for $v$ on $\mathbb{R}^{n} \backslash B\left(\mathbf{0}, d_{T}\right) \times[0, T]$ is irrelevant to the problem. Denote the solution by $Q=\cup_{0 \leqslant t \leqslant T}(\mathrm{D}(t) \times\{t\})$ and set $\Gamma(t)=\partial \mathrm{D}(t)$. Then by our analysis on the geometric problem we can find a constant $C_{1}=C_{1}\left(n, \alpha, c, \mathrm{D}_{0}\right)$ such that for all $t, s \in[0, T]$,

$$
\begin{aligned}
& \mathrm{D}(s) \subset \mathrm{D}(t), \quad|t-s| \leqslant \operatorname{dist}(\Gamma(s), x)|\leqslant c| t-s \mid, \quad \forall 0 \leqslant s<t, x \in \Gamma(t), \\
& \mathcal{H}^{n-1}(\Gamma(t) \cap B(x, \rho)) \leqslant C_{1} \rho^{n-1}, \quad \forall \rho \in\left(0, R_{*}\right], \quad R_{*}:=\frac{1}{\kappa} .
\end{aligned}
$$

Now let $w$ be the unique solution to

$$
\frac{\partial w}{\partial t}-\Delta w=\frac{\partial}{\partial t} \chi_{Q} \text { in } \mathbb{R}^{n} \times(0, T], \quad w(\cdot, 0)=0 \text { on } \mathbb{R}^{n} \times\{0\} .
$$

The estimate given in the previous section gives

$$
\begin{aligned}
& {[w]_{\gamma} \leqslant c C(n, \gamma) C_{1} R_{*}^{-\gamma / 2}=C_{2}\left(n, \alpha, \gamma, c, \mathrm{D}_{0}\right) \kappa^{\gamma / 2}, \quad \forall \gamma \in(0,1),} \\
& |\nabla w(x, t)| \leqslant K\left(n, \alpha, \kappa, c, D_{0}\right)[1+|\ln \operatorname{dist}(x, \Gamma(t))|], \quad \forall x \notin \Gamma(t), t \in[0, T] .
\end{aligned}
$$

We define $\mathbf{T} u$ as the restriction of $u_{0}+w$ on $\bar{B}\left(\mathbf{0}, d_{T}\right) \times[0, T]$ :

$$
\mathbf{T} u:=u_{0}+\left.w\right|_{\bar{B}\left(\mathbf{0}, d_{T}\right) \times[0, T]} .
$$


Then,

Finally, fix

$$
[\mathbf{T} u]_{\alpha} \leqslant\left[u_{0}\right]_{\alpha}+C_{2} \kappa^{\alpha / 2} \leqslant\left[u_{0}\right]_{\alpha}+\frac{L C_{2}^{2}}{2}+\frac{\kappa^{\alpha}}{2 L} .
$$

$$
\kappa:=2\left[u_{0}\right]_{\alpha}+L C_{2}^{2}\left(n, \alpha, c, \mathrm{D}_{0}\right) .
$$

We see that $\mathbf{T} u \in \mathbf{X}(\kappa)$. Note that $\mathbf{T} u \in C^{\gamma, \gamma / 2}\left(\bar{B}\left(\mathbf{0}, d_{T}\right) \times[0, T]\right)$ for any $\gamma \in(0,1)$ where the bound of the Hölder norm is independent of $u \in \mathbf{X}(\kappa)$. Hence $\mathbf{T}$ maps $\mathbf{X}(\kappa)$ into a compact subset of $\mathbf{X}(\kappa)$.

3. To apply the Schauder fixed point theorem, we wish to show that $\mathbf{T}$ is continuous. However this is almost impossible for the function space we are working on. To overcome this difficulty, we use a limit process by approximating $\mathbf{T}$ by $\left\{\mathbf{T}_{i}\right\}$. For each integer $i$, we define

$$
\mathbf{T}_{i} u=\rho_{i} * u_{0}+\rho_{i} * w
$$

where $*$ stands for spatial convolution, $\rho_{i}(x)=i^{n} \rho(i x)$ and $\rho(\cdot)$ is a smooth non-negative function supported on the unit ball with mass one.

For each large enough integer $i$ we work on the set

$$
\mathbf{X}_{i}(\kappa)=\left\{u \in \mathbf{X}(\kappa) \mid\|u\|_{C^{1,0}} \leqslant i^{2}\right\} .
$$

Here

$$
\|u\|_{C^{1,0}}=\|u\|_{L^{\infty}}+\|\nabla u\|_{L^{\infty}} .
$$

One can show that $\mathbf{T}_{i}$ maps $\mathbf{X}_{i}(\kappa)$ into its compact subset. Also, since solutions to the geometric problem is continuous with Lipschitz continuous speed, we see that $\mathbf{T}_{i}$ is continuous in the $C^{1,0}\left(\bar{B}\left(\mathbf{0}, d_{t}\right) \times[0, T]\right)$ topology. Thus, there exists $u_{i} \in \mathbf{X}_{i}$ such that $\mathbf{T}_{i} u_{i}=u_{i}$. As $\left\{u_{i}\right\}$ is a compact subset of $\mathbf{X}(\kappa)$, a subsequence, which we still denote by $\left\{u_{i}\right\}$, converges to a limit, say $u$, uniformly on $\bar{B}\left(\mathbf{0}, d_{T}\right) \times[0, T]$. Denote by $Q_{i}$ the solution of domain expansion with speed $V\left(u_{i}\right)$ and by $Q$ that from $V(u)$.

Note that $u_{i}=\mathbf{T}_{i} u_{i}$ satisfies $\left[u_{i}\right]_{\alpha} \leqslant \kappa^{\alpha} / L$ and

$$
\left|\nabla u_{i}(x, t)\right| \leqslant K\left(u_{0}, n, \alpha, \kappa, c, D_{0}\right)\left[1+\left|\ln \operatorname{dist}\left(x, \Gamma_{i}(t)\right)\right|\right] \quad \text { if } \operatorname{dist}\left(x, \Gamma_{i}(t)\right)>\frac{1}{i} .
$$

Then by Theorem 8 and Remark 6.1, $\chi_{Q_{i}} \rightarrow \chi_{Q}$ in $L^{1}$ so that $u_{t}-\Delta u-f=\partial_{t} \chi_{Q}$ in the distribution sense. Thus, $(u, Q)$ is the solution to our free boundary problem. This completes the proof.

REMARK 8.1 One may show that as a function of space variable, $\chi Q_{i}(x, t)=\chi_{\mathrm{D}_{i}(t)}(x)$ is a BV function so along a sequence it converges almost everywhere to a limit, say $\chi_{\hat{Q}}$. Nevertheless, in general, such a limit $\hat{Q}$ is only a very weak solution to the domain expansion problem; that is, $\hat{Q}=\lim Q_{i}$ may not be the solution to the problem of domain expansion with speed $V(u)$ starting from $D_{0}$ in the sense defined in this paper; see the Example 3. Hence, Theorem 8 is essential to the success of our last step.

REMARK 8.2 If $T=\infty$, one can step by step extend the solution to $R^{n} \times(i, i+1]$ for each integer $i=0,1,2, \cdots$. This is possible since $\kappa$ has an a priori bound (8.3), each $D(i)$ has the interior cone property (by Theorem 3), and the domain expansion problem has a semi-group property. 
Acknowledgments. The first author thanks Professor Bo Su for numerous discussions with him about his paper [34] that initiated the current work. The authors would like to thank the anonymous referee for valuable suggestions and for providing important references related to our work. This research is partially supported by the National Science Foundation grant DMS-0504691 and DMS1200599.

\section{REFERENCES}

1. Alvarez, O., Cardaliaguet, P., \& Monneau, R. Existence and uniqueness for dislocation dynamics with nonnegative velocity. Interfaces Free Bound. 7 (2005), 415-434. Zbl1099. 35148 MR2191694

2. Avrami, M. Kinetics of phase change I. General theory. J. Chem. Phys. 7 (1939), 1103-1112.

3. BARLES, G. Remark on a flame propagation model. Rapport INRIA 464 (December 1985).

4. Barles, G., Cardaliaguet, P., Ley, O., \& Monteillet, A. Uniqueness results for nonlocal Hamilton-Jacobi equations. J. Funct. Anal. 257 (2009), 1261-1287. Zbl1169. 49028 MR2541269

5. BARron, E. N., \& LIU, W. Semicontinuous solutions for Hamilton-Jacobi equations and the $L^{\infty}$-control problem. Appl. Math. Optim. 34 (1996), 325-360. Zbl0881. 49019 MR1408743

6. Brauner, C.-M., Fife, P. C., NAmah, G., \& Schmidt-Lainé, C. Propagation of a combustion front in a striated solid medium: a homogenization analysis. Quart. Appl. Math. 51 (1993), 467-493. Zb10803. 35009 MR1233525

7. Cannarsa, P., \& Cardaliaguet, P. Perimeter estimates for reachable sets of control systems. J. Convex Anal. 13 (2006), 253-267. Zbl1114.93018 MR2252231

8. Cardaliaguet, P., Ley, O., \& Monteillet, A. Viscosity solutions for a polymer crystal growth model. Indiana Univ. Math. J. 60 (2011), 895-936. Zbl1246.49026 MR2985861

9. Chen, G.-Q., \& SU, B. Discontinuous solutions for hamilton-jacobi equations: uniqueness and regularity. Discrete Contin. Dyn. Syst. 9 (2003), 167-192. Zbl1015. 35032 MR1951317

10. CHEN, X., \& HU, B. Viscosity solutions of discontinuous Hamilton-Jacobi equations. Interfaces Free Bound. 10 (2008), 339-359. Zbl1162.35019 MR2453135

11. Chen, X., \& Namah, G. Wave propagation under curvature effects in a heterogeneous medium. Appl. Anal. 64 (1997), 219-233. Zbl0878. 35009 MR1460080

12. Chen, Y. G., Giga, Y., \& Goto, S. Uniqueness and existence of viscosity solutions of generalized mean curvature flow equations. J. Differential Geom. 33 (1991), 749-786. Zb10696. 35087 MR1100211

13. Cottrell, A. H. Theoretical Structural Metallurgy. St. Martin's Press, New York, 1957.

14. Crandall, M. G., Evans, L. C., \& Lions, P.-L. Some properties of viscosity solutions of HamiltonJacobi equations. Trans. Amer. Math. Soc. 282 (1984), 487-502. Zb10543. 35011 MR0732102

15. Crandall, M. G., Ishit, H., \& LiOns, P.-L. User's guide to viscosity solutions of second order partial differential equations. Bull. Amer. Math. Soc. (N.S.) 27 (1992), 1-67. Zb10755.35015 MR1118699

16. Crandall, M. G., \& Lions, P.-L. Viscosity solutions of Hamilton-Jacobi equations. Trans. Amer. Math. Soc. 277 (1983), 1-42. Zb10599. 35024 MR0690039

17. DiBenedetto, E. Continuity of weak solutions to certain singular parabolic equations. Ann. Mat. Pura Appl. 130 (1982), 131-176. Zbl0503.35018 MR0663969

18. Evans, L. C., \& SPRUCK, J. Motion of level sets by mean curvature. I. J. Differential Geom. 33 (1991), 635-681. Zbl0726.53029 MR1100206

19. Friedman, A. Variational principles and free-boundary problems. Pure and Applied Mathematics. John Wiley \& Sons Inc., New York, 1982. A Wiley-Interscience Publication. Zbl0564.49002 MR0679313

20. Friedman, A., \& Velázquez, J. J. L. A free boundary problem associated with crystallization of polymers in a temperature field. Indiana Univ. Math. J. 50 (2001), 1609-1649. Zbl1041. 35091 MR1889073 
21. GibBs, J. W. The Collected Works of J Willard Gibbs. Yale University Press, New Haven, 1948. Zbl0031.13504

22. Giga, Y., Goto, S., \& IshiI, H. Global existence of weak solutions for interface equations coupled with diffusion equations. SIAM J. Math. Anal. 23 (1992), 821-835. Zb10754. 35061 MR1166559

23. Hohenberg, P., \& Halperin, B. Theory of dynamic critical phenomena. Rev. Mod. Phys. 49 (1977), $435-479$

24. TAylor, J.E., Cahn, J.W. \& Handwerker, C.A.. Geometric models of crystal growth. Acta metall. mater. 40 (1992), 1443-1472.

25. LAme, G., \& Clapeyron, B. Mémoire sur la solidification par refroidissement d'un globe liquide. Ann. Chimie Physique 47 (1831), 250-256.

26. Lions, P.-L. Generalized solutions of Hamilton-Jacobi equations, vol. 69 of Research Notes in Mathematics. Pitman (Advanced Publishing Program), Boston, Mass., 1982. Zb10497 . 35001 MR0667669

27. Meirmanov, A. M. The Stefan problem, vol. 3 of de Gruyter Expositions in Mathematics. Walter de Gruyter \& Co., Berlin, 1992. Translated from the Russian by Marek Niezgódka and Anna Crowley, With an appendix by the author and I. G. Götz. Zb10751.35052 MR1154310

28. Monasse, B., \& Haudin, J. M. Thermal dependence of nucleation and growth rate in polypropylene by non-isothermal calorimetry. Colloid \& Polymer Sci. 264 (1986), 117-122.

29. Osher, S., \& Sethian, J. A. Fronts propagating with curvature dependent speed: Algorithms based on hamilton-jacobi formulations. Journal of Comp. Physics 79 (1988), 12-49. Zbl0659.65132 MR0965860

30. RATAJSKi, E., \& JANESChITZ-KRIEGL, H. How to determine high growth speeds in polymer crystallization. Colloid \& Polymer Sci. 274 (1996), 938-951.

31. Rubenstein, L. I. The Stefan problem. American Mathematical Society, Providence, R.I., 1971. Translated from the Russian by A. D. Solomon, Translations of Mathematical Monographs, Vol. 27. MR0351348

32. Schulze, G., \& Naujeck, T. A growing 2 d spherulite and calculus of variations. Colloid \& Polymer Sci. 269 (1991), 689-703.

33. Soravia, P. Generalized motion of a front propagating along its normal direction: a differential games approach. Nonlinear Anal. 22 (1994), 1247-1262. Zb10814. 35140 MR1279982

34. Su, B., \& BURGER, M. Weak solutions of a polymer crystal growth model. CAM-Report 06-40, UCLA (2006).

35. SU, B., \& BURGER, M. Global weak solutions of non-isothermal front propagation problem. Electron. Res. Announc. Amer. Math. Soc. 13 (2007), 46-52 (electronic). Zbl1132. 76056 MR2320681

36. Visintin, A. Models of phase transitions. Progress in Nonlinear Differential Equations and their Applications, 28. Birkhäuser Boston Inc., Boston, MA, 1996. Zbl0882. 35004 MR1423808 